\title{
THE SWIFT GAMMA-RAY BURST HOST GALAXY LEGACY SURVEY. I. SAMPLE SELECTION AND REDSHIFT DISTRIBUTION
}

D. A. Perley ${ }^{1,2}$, T. Krühler ${ }^{2,3}$, S. Schulze ${ }^{4,5}$, A. de Ugarte Postigo ${ }^{6}$, J. Hjorth ${ }^{2}$, E. Berger ${ }^{7}$, S. B. Cenko ${ }^{8,9}$, R. Chary ${ }^{10}$, A. Cucchiara ${ }^{8}$, R. Ellis ${ }^{1}$, W. Fong ${ }^{11,17}$, J. P. U. Fynbo ${ }^{2}$, J. Gorosabel ${ }^{6}$, J. Greiner ${ }^{12}$, P. JAKObSSON ${ }^{13}$, S. Kim ${ }^{4,5}$, T. LASKAR ${ }^{7}$, A. J. Levan ${ }^{14}$, M. J. MichaŁowski ${ }^{15}$, B. Milvang-Jensen ${ }^{2}$, N. R. TAnviR ${ }^{16}$, C. C. Thöne ${ }^{6}$, And K. Wiersema ${ }^{16}$

${ }^{1}$ Department of Astronomy, California Institute of Technology, MC 249-17, 1200 East California Boulevard, Pasadena, CA 91125, USA

${ }^{2}$ Dark Cosmology Centre, Niels Bohr Institute, University of Copenhagen, Juliane Maries Vej 30, DK-2100 København Ø, Denmark; dperley@dark-cosmology.dk

${ }^{3}$ European Southern Observatory, Alonso de Córdova 3107, Vitacura, Casilla 19001, Santiago 19, Chile

${ }^{4}$ Instituto de Astrofísica, Facultad de Física, Pontificia Universidad Católica de Chile, Vicuña Mackenna 4860, 7820436 Macul, Santiago 22, Chile

${ }^{5}$ Millennium Institute of Astrophysics, Vicuña Mackenna 4860, 7820436 Macul, Santiago, Chile

${ }^{6}$ Instituto de Astrofsica de Andalucía (IAA-CSIC), Glorieta de la Astronomía s/n, E-18008, Granada, Spain

${ }^{7}$ Harvard-Smithsonian Center for Astrophysics, 60 Garden Street, Cambridge, MA 02138, USA

${ }^{8}$ Astrophysics Science Division, NASA/Goddard Space Flight Center, Greenbelt, MD 20771, USA

${ }^{9}$ Joint Space Science Institute, University of Maryland, College Park, MD 20742, USA

${ }^{10}$ US Planck Data Center, MS220-6, Pasadena, CA 91125, USA

${ }^{11}$ Steward Observatory, University of Arizona, 933 North Cherry Avenue, Tucson, AZ 85721, USA

${ }^{12}$ Max-Planck-Institut für extraterrestrische Physik, Giessenbachstrasse, D-85748 Garching, Germany

${ }^{13}$ Centre for Astrophysics and Cosmology, Science Institute, University of Iceland, Dunhagi 5, 107 Reykjavík, Iceland

${ }^{14}$ Department of Physics, University of Warwick, Coventry CV4 7AL, UK

${ }^{15}$ Scottish Universities Physics Alliance, Institute for Astronomy, University of Edinburgh, Royal Observatory, Edinburgh, EH9 3HJ, UK

${ }^{16}$ Department of Physics and Ast3ronomy, University of Leicester, University Road, Leicester, LE1 7RH, UK Received 2015 April 9; accepted 2015 October 31; published 2016 January 19

\begin{abstract}
We introduce the Swift Gamma-Ray Burst Host Galaxy Legacy Survey ("SHOALS”), a multi-observatory highredshift galaxy survey targeting the largest unbiased sample of long-duration gamma-ray burst (GRB) hosts yet assembled (119 in total). We describe the motivations of the survey and the development of our selection criteria, including an assessment of the impact of various observability metrics on the success rate of afterglow-based redshift measurement. We briefly outline our host galaxy observational program, consisting of deep Spitzer/IRAC imaging of every field supplemented by similarly deep, multicolor optical/near-IR photometry, plus spectroscopy of events without preexisting redshifts. Our optimized selection cuts combined with host galaxy follow-up have so far enabled redshift measurements for 110 targets (92\%) and placed upper limits on all but one of the remainder. About $20 \%$ of GRBs in the sample are heavily dust obscured, and at most $2 \%$ originate from $z>5.5$. Using this sample, we estimate the redshift-dependent GRB rate density, showing it to peak at $z \sim 2.5$ and fall by at least an order of magnitude toward low $(z=0)$ redshift, while declining more gradually toward high $(z \sim 7)$ redshift. This behavior is consistent with a progenitor whose formation efficiency varies modestly over cosmic history. Our survey will permit the most detailed examination to date of the connection between the GRB host population and general star-forming galaxies, directly measure evolution in the host population over cosmic time and discern its causes, and provide new constraints on the fraction of cosmic star formation occurring in undetectable galaxies at all redshifts.
\end{abstract}

Key words: galaxies: evolution - galaxies: high-redshift - galaxies: star formation - gamma-ray burst: general surveys

\section{INTRODUCTION}

Long-duration gamma-ray bursts (GRBs) are relativistic, jetted explosions of very massive stars at the end of their lives. ${ }^{18}$ The peak luminosities of GRBs and their afterglows exceed those of the most luminous supernovae, galaxies, and quasars by orders of magnitude (e.g., Bloom et al. 2009) throughout the electromagnetic spectrum. As a result, GRBs are routinely detected from great distances: the median redshift of Swift GRBs is $z \sim 2$ (Jakobsson et al. 2006; Fynbo et al. 2009b), and GRBs have been detected up to a redshift of $z=8-9$ (Salvaterra et al. 2009; Tanvir et al. 2009; Cucchiara et al. 2011).

\footnotetext{
17 Einstein Fellow.

18 They are physically distinct from short $\left(T_{90}<2 \mathrm{~s}\right)$ GRBs, which are believed to be associated with merging compact objects (Berger 2014) and are not discussed in this work.
}

GRBs are therefore fundamentally cosmological objects, and their study is intimately coupled with that of high-redshift galaxies and cosmic evolution. GRB afterglows make excellent probes of individual galaxy sightlines, a technique that has been extensively exploited to characterize the interstellar medium in distant star-forming galaxies-chemical abundances, kinematics, and dust properties - to a level of detail unmatched by any other technique (e.g., Prochaska et al. 2007, 2009; Elíasdóttir et al. 2009; D’Elia 2011; Zafar et al. 2012; De Cia et al. 2013; Thöne et al. 2013; Sparre et al. 2014; Cucchiara et al. 2015; Friis et al. 2015). They may also be useful for studies of galaxy and cosmic evolution in a broader sense. Because GRBs originate from short-lived massive stars, they stochastically sample the sites of cosmic star formation in proportion to their relative contributions to the cosmic total, providing (in principle) a means of estimating the importance of different epochs or different galaxy populations 
to the universe's stellar mass assembly (e.g., Totani 1997; Wijers et al. 1998; Blain \& Natarajan 2000; Porciani \& Madau 2001). This includes populations difficult to study by other means: very low luminosity galaxies (Berger et al. 2002; Greiner et al. 2015; Schulze et al. 2015), very dusty galaxies (Djorgovski et al. 2001; Ramirez-Ruiz et al. 2002; Berger et al. 2003; Tanvir et al. 2004; Le Floc'h et al. 2006; Michałowski et al. 2008; Perley et al. 2009c, 2013, 2015a; Greiner et al. 2011; Rossi et al. 2012; Hunt et al. 2014; Schady et al. 2014; Kohn et al. 2015), very high redshift galaxies (Lamb \& Reichart 2000; Tanvir et al. 2012; Trenti et al. 2012), and possibly even Population III stars (Bromm \& Loeb 2006; Campisi et al. 2011a).

These broader questions of using GRBs as probes of galaxy evolution and cosmology have provided major drivers for GRB research over the past two decades and in particular since 2004, when the launch of the Swift satellite (Gehrels et al. 2004) revolutionized the field with its ability to detect and instantly localize GRBs in large numbers $\left(\sim 100 \mathrm{yr}^{-1}\right)$. The vast (and still expanding) Swift legacy data set, including follow-up provided by the worldwide ground-based community, has made possible the study of GRBs, their environments, and their host galaxies in sufficiently large numbers to investigate these topics statistically.

The cosmic star formation rate (SFR) is not necessarily the only factor expected to affect the GRB rate, however. The initial chemical composition of a star influences its fate, and many models specifically predict that the GRB rate in metalpoor environments should be different-typically, higherthan in metal-rich ones (e.g., MacFadyen \& Woosley 1999; Fryer \& Heger 2005; Hirschi et al. 2005; Yoon \& Langer 2005; Woosley \& Heger 2006). Alternatively, variations in the initial mass function or close-binary fraction could affect the GRB rate (Davé 2008; Wang \& Dai 2011), as might dynamical interactions in dense clusters (van den Heuvel \& Portegies Zwart 2013). If present, these effects would have to be taken into account when attempting to employ GRBs to investigate broader cosmological issues.

A nonconstant GRB production efficiency (defined as the GRB rate relative to SFR) is supported by several studies of the GRB host population at $z \lesssim 1$ showing known GRB hosts to be smaller, bluer, less massive, and less chemically enriched on average than typical star-forming galaxies or core-collapse supernova hosts (Fruchter et al. 2006; Le Floc'h et al. 2006; Stanek et al. 2006; Modjaz et al. 2008; Levesque et al. 2010; Svensson et al. 2010; Boissier et al. 2013; Graham \& Fruchter 2013; Kelly et al. 2014; Vergani et al. 2015). Furthermore, a number of studies have concluded that the comoving GRB rate density (as inferred from the redshift distribution) evolves differently from the SFR density, showing an excess of GRBs originating from $z \gtrsim 3$ compared to what would be expected if the GRB rate was a fixed fraction of the SFR in all types of galaxy and at all redshifts (e.g., Daigne et al. 2006; Guetta \& Piran 2007; Le \& Dermer 2007; Salvaterra \& Chincarini 2007; Kistler et al. 2008; Yüksel et al. 2008).

Even so, the actual nature of the connection between the GRB rate and SFR is not yet well determined. It is not certain whether the nature of the low- $z$ population is best explained by a dependence on metallicity or on another factor that correlates with it, such as SFR intensity (Kocevski \& West 2011; Kelly et al. 2014). Other authors dispute whether the GRB rate varies significantly with host galaxy or with redshift at all (e.g., Wanderman \& Piran 2010; Campisi et al. 2011b; Elliott et al. 2012; Michałowski et al. 2012; Hunt et al. 2014; Kohn et al. 2015).

There are several complicating factors that affect these studies. Many of the best-studied hosts are those of the socalled low-luminosity GRBs that dominate the rate locally $(z<0.25)$ - but which have properties very different (orders of magnitude in energetics, intrinsic rate, and degree of beaming) from the cosmological GRBs that are detected at high redshifts (Pian et al. 2006; Guetta \& Della Valle 2007; Wiersema et al. 2007; Virgili et al. 2009; Bromberg et al. 2011). Despite their much greater observed numbers, the hosts of the distant, cosmological GRBs are more difficult to study and have been explored less consistently and in much less detail, and major selection biases underlie our ability to produce representative samples. The studies of the cosmological GRB population that have been conducted to date have in all cases been small $(<20$ objects), heavily selection biased, or characterized the hosts only minimally (observations in one to two photometric bands, often to limited depth).

In this paper we introduce and summarize the largest and most ambitious survey of the GRB host galaxy population conducted to date, designed to move past these earlier limitations by constructing a sample that is large, unbiased, and thoroughly observed at a variety of wavelengths across the electromagnetic spectrum. Our effort, which we designate the Swift Gamma-Ray Burst Host Galaxy Legacy Survey (or "SHOALS"), provides the most complete view (in wavelength, redshift, and sample construction) yet of the environments in which GRBs explode in order to rigorously examine all aspects regarding the connection between GRBs and galaxies across cosmic history. What is the GRB rate history? What is the distribution of properties among galaxies that host GRBs, how does it compare to the properties of galaxies thought to dominate the universe's star formation activity, and how does this change with redshift? What fraction of the population originates from obscured regions or from dusty galaxies? What fraction originates from galaxies too faint to be detected at all? What can we learn by combining properties measured by observing GRB afterglows in absorption (dust columns, kinematics, and gas/metal columns) with those learned by studying their hosts in emission (mass, SFR, morphology, and nebular abundance measurements)? Our survey seeks to answer these and similar questions.

This paper provides an introduction to the survey, including an explanation of our selection methodology (Sections 2-3), a summary of our observational campaign (Section 4), presentation of our new redshift determinations (Section 5), as well as tables detailing the properties of the sample. Using these observations, which establish the largest highly complete GRB redshift sample to date, we provide a measurement of the inferred cosmic GRB rate density (Section 6) unbiased by selection effects and discuss its relation to the cosmic SFR density (Section 7). We summarize these results and the project in general in Section 8.

Subsequent papers will summarize science results associated with the host galaxy campaign, an ongoing effort to provide complete spectral energy distribution (SED) measurements for 
all our targets. In particular, our Spitzer observations and the near-IR (NIR) luminosity and stellar mass distribution inferred from them will be presented in Perley et al. (2016, hereafter Paper II), which is submitted concurrently with this work. Additional papers will focus on other science questions, including the UV luminosity distribution and its evolution as inferred from the optical photometry, careful determination of the host galaxy physical properties (SFR, mass, etc.) from SED fitting and an assessment of their impact on the GRB rate, detailed analysis of correlations between properties of a host and properties of its afterglow, the spectroscopic properties of the host galaxies, an investigation into candidate hosts of foreground absorbing systems seen along GRB sightlines, and further topics.

\section{SAMPLE CONSIDERATIONS}

\subsection{Biases Affecting Redshift Measurement}

Swift detected 803 long-duration $\left(T_{90}>2 \mathrm{~s}\right)$ GRBs from the start of the mission through the end of 2014, and approximately 320 of these events have measured redshifts - a potentially enormous sample to draw from. However, from the point of view of characterizing the host population or redshift distribution, attention must be paid to systematic biases, as well as raw number statistics. In particular, the subset of Swift GRBs with measured redshift is not only incomplete (40\% of the population) but very likely to be biased: the ease of providing a redshift measurement for a GRB is expected to be dependent on properties of its host galaxy and on the redshift itself (Coward et al. 2013).

The vast majority of GRB redshifts in the Swift era are provided by absorption spectroscopy of the optical afterglow, and GRB afterglows show great variety in their optical brightnesses, especially at early times (Akerlof \& Swan 2007; Kann et al. 2010). In particular, the afterglows of some events are sufficiently faint ( $R \gtrsim 22 \mathrm{mag}$, even during the first hour) that they cannot be detected by the small- to moderate-aperture telescopes typically used to identify the optical afterglow before triggering large spectrographs (and in some cases, even deep optical imaging at large telescopes fails to detect an afterglow). These are typically referred to as "dark" GRBs (e.g., Groot et al. 1998; Fynbo et al. 2001); ${ }^{19}$ redshift catalogs based primarily on publicly reported afterglow redshifts necessarily exclude them and are therefore intrinsically optically biased. This bias removes GRBs in dusty environments or at very high redshifts, two of the most interesting regimes we might wish to use GRBs to explore, and without which our view of the redshift distribution and host population are severely incomplete.

Fortunately, there are other means available to us to recover the GRB redshifts even without an optical detection: GRB afterglows are luminous across the entire electromagnetic spectrum, and the afterglow position can often be recovered via observations at NIR, X-ray, or radio wavelengths, permitting

\footnotetext{
19 Alternatively, a GRB can be defined as "dark" based on the degree of optical faintness relative to X-ray wavelengths (e.g., via $\beta_{\mathrm{OX}}$; Jakobsson et al. 2004; van der Horst et al. 2009)—which isolates optically absorbed bursts specifically, including very luminous afterglows that can shine through thick dust screens but excluding GRBs whose faintness is intrinsic. Since this work is focused on afterglow sample completeness (and not necessarily its underlying causes), the former, "absolute" definition is generally more relevant here. We employ the term loosely to refer to GRBs with optical afterglows that are sufficiently faint that afterglow-based redshift recovery is unusually challenging or impossible.
}

the host galaxy to be identified and its redshift to be measured spectroscopically in emission (or, less optimally, photometrically). Historically, subarcsecond follow-up at wavelengths outside the optical band was much less routine than optical follow-up, and the problem was nearly intractable from a statistical point of view: dark GRB hosts and redshifts could be recovered in a few special cases (e.g., Djorgovski et al. 2001), but most of them were lost forever without an accurate localization.

This situation was transformed by Swift. The satellite is equipped with an onboard focusing X-Ray Telescope (XRT; Burrows et al. 2005) and the capability of automatically slewing to a field immediately after detecting an event with its Burst Alert Telescope (BAT; Barthelmy et al. 2005), meaning that early-time, high spatial resolution X-ray coverage is available for every event the BAT detects. XRT observations of Swift GRBs almost always detect the afterglow: 98\% of long GRBs are detected by XRT (Burrows et al. 2007), and if events for which the XRT follow-up was delayed are excluded, this figure rises to $100 \%$. So even without an optical counterpart it is almost always possible-with sufficient effort-to recover (or place a deep upper limit on) the host galaxy using the $\sim 1$ ". $5-2$ ". 0 X-ray positions routinely delivered by the XRT (Butler \& Kocevski 2007; Goad et al. 2007; Evans et al. 2009). These rapidly available positions also make it much easier to identify faint optical counterparts (or to recognize their absence and trigger deeper optical follow-up or multiwavelength observations) for bursts whose "darkness" is borderline.

Host galaxy spectroscopy (and host galaxy observations in general) is observationally expensive and can only be carried out for a limited number of objects: recovery of all missing Swift redshifts is unfeasible. Moreover, absorption redshifts can be missed for a variety of reasons other than intrinsic darkness: GRBs occur at random times and directions and some cannot be followed if, for example, the location is too close in projection to the Sun or the Galactic plane. Indeed, optical afterglows and redshifts of about half of Swift GRBs are probably missed for mundane reasons. Several studies have estimated an intrinsic dark fraction of about 20\% (Cenko et al. 2009b; Greiner et al. 2011; Melandri et al. 2012), so 80\% of Swift GRBs should have afterglows bright enough in principle for optical spectroscopy, yet in only $30 \%$ of cases are redshifts actually reported. Expending effort and observational resources to recover hosts and redshifts for events missed for these reasons would not be informative.

At the same time, for the reasons discussed above, we cannot simply neglect dark GRBs without afterglow redshifts without also discarding obscured or high- $z$ events from the sample. Two general approaches are available to characterize the complete population. The first is to simply take the known- $z$ population and attempt to "correct" it by also observing a limited number of GRBs that are definitely dark (have deep, early optical limits) and combining the samples. This has been adopted in the past (e.g., Krühler et al. 2011; Rossi et al. 2012; Perley et al. 2013; Hunt et al. 2014) and, among other things, has clearly shown that GRBs with very faint optical afterglows probe a different (dustier and more massive) host population from GRBs with bright optical afterglows. However, the actual contribution of dark GRBs to the whole is not precisely determined, in part because the matter is much more complicated than an either/or distinction between "bright" and "dark" bursts: the distribution of afterglow brightnesses is a 
continuum, as is (we expect) the likelihood of redshift measurement as a function of brightness. "Very" versus "somewhat" dark bursts may likewise have different host and redshift distributions.

An alternative approach, and the one we adopt in this work, is to carefully down-select the Swift sample to remove GRBs that occurred under circumstances that were not optimal for ground-based follow-up and isolate a subset for which the afterglow redshift completeness is close to the expected maximum achievable value of about $80 \%$ (the remaining $20 \%$ being dark bursts). This basic technique was first exploited to study unbiased afterglow demographics and redshift distributions (Jakobsson et al. 2006; Cenko et al. 2009b; Fynbo et al. 2009b; Perley et al. 2009c; Greiner et al. 2011) and more recently has been successful in addressing the properties of the GRB host galaxy population as well. Most notably, the multiyear Very Large Telescope (VLT) based "TOUGH" project (The Optically Unbiased GRB Host Survey; Hjorth et al. 2012) used a series of observability cuts to isolate a sample of 69 objects out of the broader Swift sample and has achieved a redshift completeness of close to $90 \%$ after intensive optical spectroscopy of the host galaxies of those events lacking bright afterglows (Jakobsson et al. 2012; Krühler et al. 2012; Schulze et al. 2015). "BAT-6" (Salvaterra et al. 2012) is an effort with a similar size, design, and redshift completeness (58 objects at $\gtrsim 90 \%$ completeness).

Though these projects have been informative in charactering the host population in various ways (e.g., Michałowski et al. 2012; Milvang-Jensen et al. 2012; Schulze et al. 2015; Vergani et al. 2015) and have provided the first nearly unbiased estimates of the redshift distribution (Jakobsson et al. 2012), from the perspective of measuring actual redshift evolution of the GRB host population, TOUGH and BAT-6 are limited by their modest sample sizes. For example, in the case of TOUGH, the 69 events span a redshift range of $0<z<6.3$, over which an enormous degree of cosmic evolution has occurred: to provide a snapshot of the host population at a particular epoch, and compare to other epochs, would require splitting the sample into at least five to six redshift bins, each of which would have only a few host galaxies. This is enough to make broad statements, but less than necessary to characterize the parameter distribution at any point in history.

Larger samples are therefore needed. In principle, this could simply be done by extending the year cutoff affecting earlier samples closer to the present (e.g., TOUGH was limited to events from 2005 to 2007 based on the time the survey was conducted; this cutoff could be extended to include more recent bursts). However, we can now do better: while the detailed selection criteria for earlier samples were developed based on well-informed guessing about the factors that affect the success rate of afterglow follow-up, we now have the benefit of hindsight in the form of a large Swift GRB population from which to establish in practice what parameters and values maximize the benefit to our science goals.

As a result, before proposing for and executing our survey, we devoted significant attention to examining the impact of a variety of criteria on the size and completeness level of the resulting sample, deciding on a final set of optimized parameters based on these investigations. As the associated findings are relevant to understanding the design of our survey and may also be useful to investigators considering even more ambitious projects in the future, we describe these in detail in the next section.

\subsection{Additional Considerations}

Since it is essential to produce an unbiased population, we consider only observational criteria that are not expected to be physically connected to the burst's environment in any way. Given the cosmological nature of GRBs, factors related to time or sky location are all clearly unconnected to the GRB host environment and therefore fair to consider. Properties intrinsic to the GRB itself (measurements of the prompt emission or afterglow) are less obvious. Factors specific to the prompt emission should not be biased with respect to environment, since in the favored internal/external shock paradigm (e.g., Sari \& Piran 1997) the physical mechanism producing the GRB prompt emission is unrelated to the nature of the environment it formed in. ${ }^{20}$ On the other hand, the GRB afterglow is produced by interaction of the GRB ejecta with the circumprogenitor environment (Mészáros \& Rees 1997), and its light may be attenuated by gas and dust within its host galaxy, so we do not consider any afterglow-related property.

Because our selection cuts are developed a posteriori, it is important to note that even the application of nominally unbiased cuts can produce some degree of bias in a discrete sample, depending on how many features are considered and how precisely fine-tuned the cut threshold is optimized. If the cuts are drawn from a large potential parameter space and optimized directly against the data with no separate training set, the choice of cut parameters and their values may become driven by stochastic effects-artificially driving up the completeness and reintroducing biases associated with redshift measurement. To minimize this risk, we only examine features for which a link between the variable and redshift completeness is at least potentially expected from fundamental considerations, and only cut on features where significant dependence is observed. We also only consider round-number, discrete thresholds in determining the cut value. (An alternative would have been to divide the sample into a training set and the applied sample, but as the number of known-redshift GRBs remains modest - a few hundred - this would overly restrict the sample size of both sets.)

Choosing how many cuts to apply, and how stringently to cut, also requires striking a balance between redshift completeness and overall statistical size. Even when starting with an initial sample of many hundreds of Swift events, discrete cuts are necessarily blunt and probabilistic tools, and many knownredshift events will be lost in trying to increase the completeness significantly from its very low initial value of $30 \%$. We set as a reasonable goal obtaining at least 100 events and at least $65 \%$ initial (pre-host-follow-up) redshift completeness. The target sample size is needed to provide a significant expansion over earlier host surveys and to ensure that the sample is large enough such that, even if subdivided into five or more redshift bins, there are enough targets per bin to reasonably statistically constrain the parameter distribution. The completeness goal is informed largely by the success of TOUGH in increasing its redshift completeness from a similar starting level to its current $\sim 90 \%$, which is about the level that

\footnotetext{
20 This does assume that long GRBs all represent a single class of object, rather than a superposition of multiple types with different progenitors and prompt-emission properties.
} 

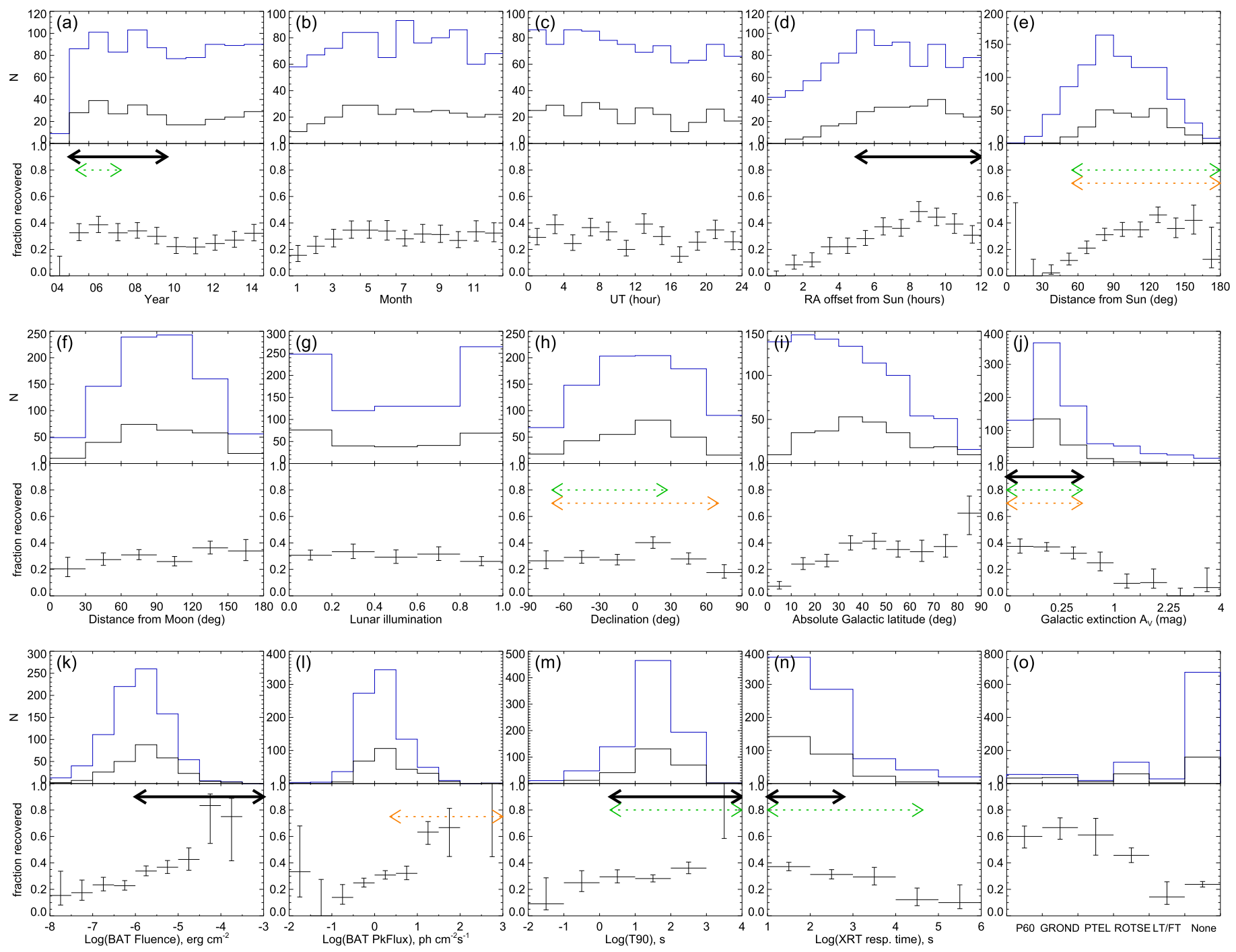

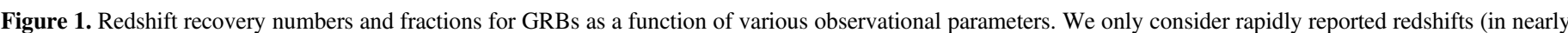

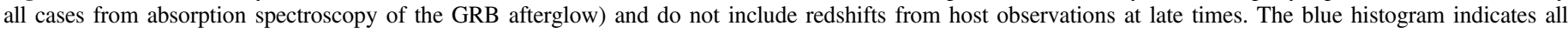

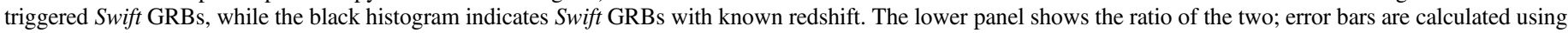

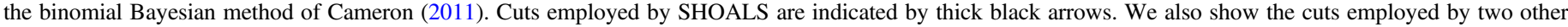
host-oriented uniform samples: TOUGH (green arrows) and BAT-6 (orange arrows).

is necessary for systematic considerations (preferentially missing redshift measurements of faint host galaxies) not to dominate the statistical ones. (We had no trouble meeting these goals, with our final cuts producing a sample of 119 events at pre-host redshift completeness of $68 \%$.)

Redshift completeness is not the only motive for applying cuts to the Swift sample-it is also desirable to maximize overlap with existing observations of GRBs and their hosts. High-quality early afterglow observations, even if not leading to a successful redshift measurement, can produce a more secure host identification, a redshift upper limit, and useful complementary information about the GRB sightline; previous host galaxy observations reduce the observational demands needed to complete the survey.

Because our Spitzer/IRAC campaign (Section 4.1) was conducted during Cycle 9 (2012 November through 2013 September), our targets were necessarily restricted to bursts occurring before that period, so we only considered events up to the end of 2012 October for inclusion.

\section{SAMPLE SELECTION AND ANALYSIS OF FACTORS INFLUENCING REDSHIFT COMPLETENESS}

With the above considerations in mind, we investigated the impact of many different observables to identify what combination of unbiased cuts would produce the highest final redshift completeness. These are explained in detail in the following subsections, with the results summarized in Figure 1. Our data were drawn from the Swift GRB table, ${ }^{21}$ supplemented by our own corrections to redshifts or classifications where appropriate, using published sources and our own observations.

For the purposes of this analysis we did not treat redshifts that were determined at late times via host galaxy observations as "known," since the latter subset may be affected by conscious (and potentially biased) follow-up considerations. We did, however, consider emission-line redshifts that were promptly reported in the GCN circulars. (For low-redshift, lowluminosity GRBs the host galaxy may be of comparable or

\footnotetext{
${ }^{21}$ http://swift.gsfc.nasa.gov/archive/grb_table/
} 
greater brightness than the optical afterglow by the time the latter is observed, so an emission-based redshift may be reported even if an absorption redshift may have been possible in absence of host emission lines.)

We consider only GRBs detected on board the satellite, excluding ground or slew-survey triggers (which are distributed only after many hours' delay and are rarely followed).

\subsection{Time of Explosion}

The amount and quality of observational follow-up directed toward a given GRB afterglow are affected by terrestrial timescales in many ways. Observatories are geographically clustered (most large optical facilities are located in Hawaii, the western United States, Chile, or the Canary Islands), and the time of day a burst explodes (as well as its location) affects which observatories can follow it and how quickly. Seasonal weather patterns also influence the likelihood of whether a given facility is able to follow up a GRB (and, if so, how effectively). On longer timescales, other factors may come into play: interest in GRB follow-up among different observational groups has both waxed and waned over Swift's lifetime, while ground-based facilities important to GRB follow-up have been commissioned (e.g., GROND, RATIR, GTC, X-shooter) and decommissioned (e.g., PAIRITEL, LOTIS) over the same period.

In Figures 1(a)-(c) ${ }^{22}$ we plot the redshift completeness as a function of calendar year, calendar month, and UT hour. While the trends are weak, at least some clear signatures are evident. First, the redshift completeness peaked in the early years of Swift (40\% between 2005 and 2009) but has fallen since then (27\% since 2010), probably because follow-up of "routine" bursts has become less common over the years and also because of smaller allocations of time to rapid-response spectroscopy. This trend is large enough that a year cut significantly improves the redshift completeness of the sample; restricting ourselves to older bursts also has the benefit that the large amount of time elapsed has provided more opportunity for previous host galaxy campaigns to acquire ground-based data. We therefore restrict the base sample to events in the fiveyear period 2005-2009. (Some post-2009 events are later included under a separate criterion; see Section 3.7.)

A seasonal effect may also exist; the redshift completeness drops significantly between January and March (26\%) compared to the rest of the year $(35 \%)$. We investigated whether this dependence may be different for northern and southern hemisphere bursts and found the trend to be present in both data sets. Despite the strength of this effect, we elected not to cut on it, since the complementary benefits are less clear than for the other cuts employed. Nevertheless, considering the impact observed despite the crudeness of a raw seasonality cut, the trend we observed here provides good reason to consider more specific cuts, based on actual weather conditions at various sites, in the future.

We see no clear secular dependence on the time of day and did not cut on this parameter.

\footnotetext{
22 Figure 1 shows all GRBs through the end of 2014 in order to provide the most up-to-date view of the population, although our selection criteria were developed in late 2012. The trends are the same in either case.
}

\subsection{Sun Angle}

Ideally, Swift's pointing would always be centered close to $180^{\circ}$ away from the Sun, such that every burst detected by the BAT could be followed immediately by any telescope in the night hemisphere of Earth and remain above the horizon until sunrise. In reality, operational considerations (in particular, the cooling requirements of the XRT) require that the pointing direction of Swift is frequently far from this ideal and many GRBs are detected close in projection to the Sun, making ground-based follow-up challenging or impossible.

In Figures 1(d)-(e) we plot the redshift completeness against angular separation from the Sun as well as against separation in right ascension only. It is clear that Sun angle has a large impact on redshift completeness, with $\sim 40 \%$ of bursts in the anti-solar hemisphere successfully recovered but extremely poor completeness for bursts nearest the Sun $(<10 \%$ within $60^{\circ}$ ).

Previous surveys (TOUGH, BAT-6) used a Sun distance cut of $>55^{\circ}$ and no right-ascension cut. However, based on the plots above, it is clear that somewhat more stringent cuts can significantly further improve the redshift completeness with only modest additional down-selection: the redshift efficiency remains very poor for events closer than $\sim 75^{\circ}$ or for bursts with a right-ascension difference of less than $5 \mathrm{hr}$. We employ a minimum right-ascension separation of $5 \mathrm{hr}$, and in principle an even more stringent cut could have been used at the expense of reducing the sample size. We make no formal cut on angular separation, but note that the right-ascension cut alone excludes all GRBs closer than $54^{\circ}$ from the Sun.

\subsection{Lunation and Moon Angle}

While the location of the burst relative to the Sun has a large impact on the ability to follow up a burst, lunar considerations appear to be negligible. In Figures 1(f)-(g) we plot the recovery rate against lunar illumination and moon distance, respectively. We see only weak, low-significance trends in either case, and so we do not use lunar information to restrict our sample. (While this result may seem surprising, we note that bursts very close to the full Moon are also very nearly anti-Sun, and the improved observability may counteract the higher sky background. We also note that bursts very close to the Moon are automatically excluded from our sample, though not from Figure 1, on the basis of the lack of XRT follow-up.)

\subsection{Declination}

Bursts that occur close to the celestial equator can be observed by telescopes in both hemispheres, whereas those occurring closer to the poles are difficult or impossible to follow from the opposing hemisphere and (in extreme cases) cannot be observed by equatorial-mount telescopes at all. On the other hand, high-declination bursts remain above the horizon for longer than equatorial ones. In practice, the declination dependence of redshift completeness appears to be weak (Figure 1(h)): no significant declination trend is seen in the southern hemisphere, and while a fairly strong trend is evident in the northern hemisphere, it becomes much less significant after our preferred $A_{V}$ and $T_{90}$ cuts (Sections 3.53.6) are applied, indicating that it may be coincidental. We therefore did not apply a declination cut. 


\subsection{Galactic Latitude and Extinction}

The position of a burst as projected against the plane of our own Galaxy strongly impacts our ability to recover its optical afterglow, mostly as a result of extinction by interstellar dust within the Milky Way (but crowding and source confusion with foreground stars may also play a role). This effect can be clearly seen in Figures 1(i) and (j), where the recovery fraction is plotted against both the absolute Galactic latitude $|b|$ and the foreground extinction $A_{V}$ (as calculated from the Schlegel et al. 1998 dust maps ${ }^{23}$ ). For extinction columns in excess of $\gtrsim 0.5 \mathrm{mag}$, and for latitudes less than $|b| \lesssim 10^{\circ}$, the recovery fraction plummets.

We employ a maximum foreground extinction of $A_{V}<0.5 \mathrm{mag}$ (Schlegel) in SHOALS, which of course in practice also cuts most events near the Galactic plane. This is the same criterion used by both TOUGH and BAT-6.

\subsection{Prompt Emission Properties: Fluence, Flux, and Duration}

The GRB prompt emission is not thought to depend directly on the properties of the circumprogenitor environment, so incorporating prompt-emission properties in our selection should not bias the sample-although such selections will necessarily impact properties such as the intrinsic redshift distribution of the sample versus that of the Swift parent population (we expect brighter bursts to be closer, on average). More importantly, GRB prompt-emission brightness is also observed to be correlated with GRB afterglow brightness (Gehrels et al. 2008; Nysewander et al. 2009; Kann et al. 2010), partly because luminous bursts tend to release more kinetic energy into their surroundings and produce brighter afterglows, and also simply because closer bursts (at fixed luminosity) will be brighter at all wavelengths. We therefore expect that the brighter afterglows associated with a population of bright GRBs should make their redshifts easier to measure.

The impact of adding a prompt-emission cut on redshift completeness was first clearly demonstrated by Salvaterra et al. (2012): the BAT-6 sample reached $\sim 80 \%$ redshift completeness even without any host galaxy follow-up after application of a fairly stringent cut on the peak photon flux in addition to their observability criteria (after host follow-up this rose to $\sim 90 \%$ ). A flux/fluence cut is also desirable from the point of view of better understanding events with optical nondetections, since it is easier to establish limits on the spectral index $\beta_{\mathrm{OX}}$ (Jakobsson et al. 2004; van der Horst et al. 2009) and determine whether the afterglow is obscured in the presence of a brighter X-ray afterglow. Finally, imposition of an explicit cut on prompt emission properties serves to replace the complicated Swift triggering criteria (Band 2006) with a simple well-defined threshold that can be more easily modeled for the purpose of measuring intrinsic rates and redshift/luminosity distributions (Section 6.1). ${ }^{24}$

\footnotetext{
${ }^{23}$ We use the original Schlegel et al. maps in preference to more recent work (e.g., Peek \& Graves 2010; Schlafly \& Finkbeiner 2011) for consistency with earlier GRB host selection efforts.

${ }^{24}$ We wish to emphasize that cutting on fluence may in principle affect the redshift distribution - but does so in a controlled way that improves, rather than harms, our ability to accurately measure the intrinsic burst rate. The BAT sample is always flux/fluence limited; our cut replaces the complex on-board trigger criteria with a well-defined selection criterion that we can correct for. This issue is discussed further in Section 6.2.
}

We plot the dependence of the redshift completeness on the BAT $15-150 \mathrm{keV}$ energy fluence and $1 \mathrm{~s}$ peak photon flux in Figures 1(k)-(l). The redshift completeness rises sharply for brighter bursts. Both fluence and peak flux show trends of similar magnitude, although in practice we found that using a fluence cut produced a slightly higher redshift completeness: after all other cuts were applied, the redshift completeness was $4 \%$ higher for a fluence cut than for a peak flux cut chosen to produce the same final sample size. A fluence cut is also expected to affect the redshift distribution less than a peak-flux cut would, since the cosmological dimunition in luminosity is offset by time dilation (see also Section 6.1). As a result, we choose to cut on fluence $\left(S_{15-150 \mathrm{keV}}>10^{-6} \mathrm{erg} \mathrm{cm}^{-2}\right)$.

We remove events with a prompt-emission duration of less than two seconds from the sample, requiring $T_{90}>2 \mathrm{~s}$. This was done not for its effects on redshift completeness, but rather to remove short-duration GRBs from the sample, as these appear to have a physically distinct origin associated with compact object mergers and a very different host population (e.g., Fong et al. 2013). Strictly, this cut actually has no impact: our fluence cut strongly disfavors short events and removes all events with $T_{90}<2 \mathrm{~s}$ on its own. However, we also remove two events with $T_{90}>2 \mathrm{~s}$ (GRB 060614 and GRB 080503) whose prompt-emission light curves resemble those of short-duration events with extended emission (while their exact origins are debated, these events have properties very different from ordinary long-duration GRBs; see, e.g., Fynbo et al. 2006; GalYam et al. 2006; Gehrels et al. 2006; Perley et al. 2009b for further discussion).

Unlike the other properties discussed in this section, the BAT measurements are subject to measurement uncertainties, and in principle values can change upon reanalysis. Our selection was conducted using values in the Swift GRB table, which is collated from values reported in the BAT refined analysis GCN circulars (which in turn are based on analysis conducted by the BAT team 1-2 days after the event). Further analysis and improved measurements have been carried out only for a subset of these (e.g., Sakamoto et al. 2011). Since we wished to examine the entire Swift sample using the same data, we used the GCN values to establish our selection. Fortunately, the differences are usually very small (a few percent), and the choice has negligible impact on the sample properties. Only four new GRBs would have entered the sample had we used the updated values on bursts where they are available: GRB 060605 at $z=3.78$ (Ferrero et al. 2009), GRB 060124 at $z=2.300$ (Fynbo et al. 2009b), GRB 090102 at $z=1.547$ (de Ugarte Postigo et al. 2009c), ${ }^{25}$ and GRB 090728 (unknown-z; possibly an $R$-dropout and at $z \sim 6$; Melandri et al. 2009). No GRBs currently in the sample would be dropped from it.

\subsection{Rapid Observability to Swift}

Some events that the Swift BAT detects cannot be rapidly observed by the XRT owing to pointing considerations or technical difficulties. In such cases, the lack of a prompt X-ray position reduces some of Swift's advantages relative to previous satellites. Optical afterglow follow-up is more difficult because observers must identify an (often faint) new source within the full $3^{\prime}$ radius initial BAT error circle, potentially causing afterglow identifications to be delayed or missed

\footnotetext{
25 The change in status for GRB 090102 is not due to reanalysis but is the result of a typo in the Swift GRB table.
} 
entirely and resulting in lower redshift completeness (a trend that can be seen in Figure 1(n)). Furthermore, because latertime X-ray observations do not guarantee a detection, host galaxy follow-up and redshift identification may be strictly impossible in some cases if early observations are not carried out. For both these reasons, we require a prompt XRT response $(<600 \mathrm{~s})$.

\subsection{Rapid Observability to Specific Ground Facilities}

Many of the factors described in earlier sections (Sun angle, etc.) are chosen based on their impact on ground-based observability. Of course, other factors we have not explicitly considered also affect whether or not a ground-based telescope can actually respond to a burst, including weather conditions, the instrument set(s) available that night, and whether or not the telescope was under maintenance.

In principle, it would be desirable to explicitly incorporate site-dependent weather and telescope downtime at the most important facilities into our calculations as a formal sample cut. This may be a promising avenue for establishing future samples (in particular, for more recent bursts, given the lower degree of redshift completeness overall since 2010), but as this information is not readily available, we did not consider it in designing our survey. However, we can be even more precise by considering whether or not several leading ground-based imaging response facilities actually were successfully triggered by Swift for early-time observations. Such an approach has already been applied extensively by our team and others to produce uniform optical afterglow samples (Cenko et al. 2009b; Perley et al. 2009c; Greiner et al. 2011), and the same procedure can be applied to GRB hosts.

Figure 1(o) shows the recovery rate for events observed "rapidly" by various GRB-oriented follow-up facilities: the Palomar 60-inch telescope (P60, bursts from 2005 to 2012; Cenko et al. 2009b; work in preparation), the Gamma-Ray Burst Optical/Near-infrared Detector (GROND, 2007-2012; Greiner et al. 2011; T. Krühler et al. 2015, private communication), the Peters Automated Infrared Telescope (PAIRITEL, 2005-2010; Morgan 2014), the Robotic Optical Transient Search Experiment (ROTSE-III, 2005-2012; taken from a search of the GCN circulars), and the combination of Faulkes and Liverpool telescopes (2005-2007; Melandri et al. 2008), compared to events observed by none of these facilities on a rapid timescale. Except in the case of the (small) Liverpool/Faulkes sample, the triggering of early-time observations is, unsurprisingly, correlated with modest increases in recovery rate.

Except possibly in the case of the ROTSE events (whose redshift completeness is lower than desired), these samples are not large enough for our purposes, even if applied with no other cuts. Therefore, instead of requiring ground-based follow-up by one of these facilities, we treat rapid observations to either P60 or GROND as an alternative criterion, allowing these bursts to bypass the three afterglow-observability requirements (year, hour angle, and XRT-response time), such that a GRB nominally failing one or more of these criteria can nevertheless enter the sample as long as either of these two telescopes observed it at early times. GROND or P60 observations, however, do not provide immunity to criteria not connected with ground observability, in particular, the fluence and extinction cuts that remain in effect across the entire sample.
To maximize overlap with the TOUGH sample (Hjorth et al. 2012), for which extensive host galaxy data are already available, we offer a similar bypass to events within that sample (but, again, with the requirement that they must still satisfy the fluence cut).

\subsection{Additional Host Observability Criteria}

Two additional criteria are added to ensure that host galaxy follow-up is possible. First, we require that a $<2^{\prime \prime}$ (at $90 \%$ confidence) position be available, since uniquely identifying a host galaxy in an area larger than this carries significant risk of misidentification or ambiguity. Because all of our events were observed by the XRT at early times when the burst was bright (or by a sensitive ground-based optical facility), a position of this accuracy is almost always available, and this cut removes only a single event (GRB 080613B) from consideration. In principle, this exclusion creates a very weak bias (if this event had an optical afterglow, we would have included it; two other events, GRB 071021 and GRB 061110B, have >2" XRT position uncertainties but were included thanks to available optical/IR follow-up)_but given its small impact in practice, we ignore this effect.

We also require that the host position not be contaminated by a bright foreground object in the form of a Galactic star or intervening galaxy. This decision is somewhat subjective (stars and galaxies contaminate many fields to various degrees, especially in the Spitzer imaging due to its large point-spread function $[\mathrm{PSF}]$ ) and in the case of a contaminating galaxy requires some degree of deep follow-up to have been obtained in the first place to recognize that the foreground galaxy is not the host. Events that we exclude on the basis of a contaminating bright star are GRB 050716, GRB 060923C, GRB 071003, GRB 080129, GRB 080212, GRB 080229A, GRB 080905B, and GRB 101023A. Events excluded as a result of a contaminating galaxy are GRB 080319C (for which ground-based spectroscopy and Hubble Space Telescope [HST] imaging show the source closest to the afterglow location to be a superposition of the host and a foreground system at $z=0.81$ ) and GRB 081028 (for which no ground-based imaging or spectroscopy is available, but for which the observed IRAC magnitude of the source underlying the afterglow position is inconsistent with any galaxy at that redshift, so it is probably one of the foreground absorbers mentioned in Berger et al. 2008).

\subsection{Summary and Sample Properties}

We summarize our final selection criteria $^{26}$ in Table 1. Characteristics of the sample are presented in Table 2, and a table of key properties of the sample relevant to our study (positions and redshifts; see subsequent sections) is presented in Table 3.

As these numbers indicate, our selection criteria were highly successful in isolating a large, well-observed sample of Swift GRBs: 119 targets, $68 \%$ of which had redshifts measured before any late-time host follow-up had been conducted. Including redshifts from late-time follow-up (both preceding

\footnotetext{
${ }^{26}$ While final for the purposes of this study, our sample is readily extendable by loosening or expanding the afterglow observability criteria. In hypothetical future cases where it becomes necessary to be specific, we will refer to the uniform sample established by the specific criteria outlined in Table 1 as the SHOALS09+ sample. In this paper we will simply refer to it as the SHOALS sample.
} 
Table 1

Summary of Selection Criteria

\begin{tabular}{|c|c|c|c|c|}
\hline No. & Type & Short Description & $\Delta N^{\mathrm{a}}$ & $\Delta C^{\mathrm{b}}$ \\
\hline 1 & GRB properties & Onboard Swift/BAT trigger before 2012 October w/XRT observations & $\ldots$ & $\ldots$ \\
\hline 2 & GRB properties & $T_{90}>2 \mathrm{~s}$ and not an $\mathrm{SGRB}+\mathrm{EE}^{\mathrm{c}}$ & -2 & $+0.4 \%$ \\
\hline 3 & GRB properties & $S_{15-150 \mathrm{keV}}>10^{-6} \mathrm{erg} \mathrm{cm}^{-2}$ & -69 & $+9.6 \%$ \\
\hline 5 & Afterglow visibility & Amenable to follow-up: (5a-i and 5a-ii and 5a-iii), OR 5b, OR 5c & -143 & $+17.1 \%$ \\
\hline $5 \mathrm{a}-\mathrm{i}$ & Afterglow visibility & XRT observations within 10 minutes & -10 & $+2.2 \%$ \\
\hline 5a-ii & Afterglow visibility & Between 2005 and 2009 (inclusive) & -36 & $+8.1 \%$ \\
\hline $5 \mathrm{c}$ & Afterglow visibility & Satisfies TOUGH criteria (Hjorth et al. 2012) & +11 & $-1.4 \%$ \\
\hline 6 & Host/afterglow visibility & No known foreground star or galaxy contaminating the position & -7 & $+2.2 \%$ \\
\hline 7 & Host visibility & $<2^{\prime \prime}$ position available & -1 & $+0.6 \%$ \\
\hline
\end{tabular}

Notes.

${ }^{a}$ Number of GRBs affected by this criterion, if applied after all other criteria; i.e., these numbers indicate the (negative of) the change in sample size if the criterion in question were removed and the sample reconstructed using all other criteria still in place. In the case of criteria $5 \mathrm{~b}$ and $5 \mathrm{c}$, numbers are positive since these criteria enable GRBs to be included despite failing one of the 5a criteria.

${ }^{\mathrm{b}}$ Increase in redshift completeness after cutting on this criterion, if applied after all other criteria. This indicates (the negative of) our change in pre-host follow-up redshift completeness if the given criterion was dropped.

${ }^{c}$ A short-duration GRB with extended emission. Only GRB 080503 and GRB 060614 fall under this category among targets not cut by other criteria.

Table 2

Sample Characteristics

\begin{tabular}{ll}
\hline \hline Statistic & Value \\
\hline Total sample size & 119 \\
Number of "early" redshifts & $81(68 \%)$ \\
Number of redshifts to date & $110(92 \%)$ \\
Number with redshift limits & $118(99 \%)$ \\
Mean redshift & 2.18 \\
Redshift quartiles & $1.26,2.06,2.77$ \\
Redshift range & $0.03-6.29$ \\
\hline
\end{tabular}

our efforts and including our host galaxy campaign; see Section 5.1), we have achieved a completeness close to $90 \%$ (89\% considering only secure spectroscopic redshifts, $92 \%$ including photometric redshifts, 94\% including $N_{H}$-bracketed and a tentative single-line spectroscopic redshift, and $99 \%$ including upper limits) so far on a sample twice the size of previous efforts. Forthcoming observations will likely increase the completeness even further.

Approximately $20-30$ events ( $\sim 20 \%$ of the sample) can be classified as "dark," with the exact number depending on the definition employed. Twenty-five GRBs have no unambigous optical $(0.3-1.0 \mu \mathrm{m})$ afterglow detection reported in the GCN circulars or elsewhere, and in only four of these cases could this be readily attributed to lack of deep or early follow-up (see Table 3 for details). On the other hand, 12 events with optical detections have red colors indicative of significant dust attenation, in some cases a great deal of dust attenuation (e.g., $A_{V} \sim 3-4 \mathrm{mag}$ for GRB 080607, GRB 090709A, and GRB 100621A; Cenko et al. 2010; Perley et al. 2011; Greiner et al. 2013) - these events probably lie in the same physical class as optically undetected dark bursts, but optical detections were secured thanks to particularly efficient follow-up and/or a very intrinsically luminous afterglow.

Since the optical afterglow observations for many of the GRBs in our sample have not been thoroughly analyzed beyond the quick reports given in the GCN circulars, it is of course possible that some additional events are (modestly) obscured without us being aware of it. Nevertheless, as our obscured fraction is consistent with other recent estimates (Perley et al. 2009c; Greiner et al. 2011; Covino et al. 2013), it is likely that the events we have identified constitute the large majority of all events that were dust obscured in our sample. The afterglow properties of the sample (including quantitative metrics of darkness such as $\beta_{\mathrm{OX}}$; Jakobsson et al. 2004) will be revisited in more detail in forthcoming papers.

\section{OBSERVATIONS AND DATA ANALYSIS}

A primary goal of our survey is to produce high-quality, multifilter SEDs for all host galaxies within the sample, enabling the construction of rest-frame luminosity functions at any wavelength and the measurement of important physical parameters (mass, SFR, etc.) via SED fitting. These efforts are still ongoing and will be described in full in subsequent papers. Instead, we briefly outline our general observational strategy and its motivations.

\subsection{IRAC Observations}

Spitzer observations are key to our effort, extending the wavelength coverage by a factor of two relative to previous host galaxy surveys and providing access to physically distinct information from what is possible from ground-based observations alone. IRAC's capabilities in its shortest-wavelength filters $(3.6$ and $4.5 \mu \mathrm{m})$ are undiminished even in its warm mission, and the instrument remains sufficiently sensitive to detect typical galaxies out to $z \sim 5$. Furthermore, the luminosity of a galaxy at these wavelengths (which always probe wavelengths redward of the Balmer break across this redshift range) is determined primarily by a single parameter (its stellar mass) with only modest dependence on age and extinction-providing a means of directly interpreting IRAC observations even without the supporting data we are amassing. (This contrasts with the situation at the rest-frame UV wavelengths probed by ground-based optical imaging, since a 
Table 3

The SHOALS GRB Sample

\begin{tabular}{|c|c|c|c|c|c|c|c|c|c|c|c|c|}
\hline GRB & $S_{\mathrm{BAT}}^{\mathrm{a}}$ & R. A. ${ }^{\text {b }}$ & Decl. $^{\text {b }}$ & Unc. $^{\mathrm{c}}$ & Type. $^{\mathrm{d}}$ & OT? ${ }^{\mathrm{e}}$ & Dusty? ${ }^{f}$ & References $^{\mathrm{i}}$ & $z$ & Early? $^{\mathrm{g}}$ & Origin $^{\mathrm{h}}$ & References $^{\mathrm{i}}$ \\
\hline 050128 & 51 & $14: 38: 17.68$ & $-34: 45: 55.4$ & 1.70 & $X$ & & & 1 & $<5.5$ & $\mathrm{~N}$ & host & * \\
\hline 050315 & 32 & $20: 25: 54.17$ & $-42: 36: 02.2$ & 0.28 & $\mathrm{O}$ & $\mathrm{Y}$ & & 2 & 1.9500 & $\mathrm{Y}$ & AG & 3 \\
\hline 050318 & 11 & 03:18:51.01 & $-46: 23: 44.0$ & 0.60 & $\mathrm{O}$ & $\mathrm{Y}$ & & 4 & 1.4436 & $\mathrm{Y}$ & $\mathrm{AG}$ & 3 \\
\hline 050319 & 13 & $10: 16: 47.94$ & $+43: 32: 53.5$ & 0.40 & $\mathrm{O}$ & $\mathrm{Y}$ & & 5 & 3.2425 & $\mathrm{Y}$ & $\mathrm{AG}$ & 6 \\
\hline 050401 & 83 & $16: 31: 28.80$ & $+02: 11: 14.0$ & 0.43 & $\mathrm{O}$ & $\mathrm{Y}$ & $\mathrm{Y}$ & 2 & 2.8983 & $\mathrm{Y}$ & $\mathrm{AG}$ & 6 \\
\hline $050525 \mathrm{~A}$ & 151 & $18: 32: 32.59$ & $+26: 20: 22.3$ & 0.31 & $\mathrm{O}$ & $\mathrm{Y}$ & & 2 & 0.606 & $\mathrm{Y}$ & host & 7 \\
\hline 050726 & 20 & 13:20:11.95 & $-32: 03: 51.2$ & 0.32 & $\mathrm{U}$ & $\mathrm{Y}$ & & 2 & $<3.5$ & $\mathrm{~N}$ & $\mathrm{AG}$ & * \\
\hline 050730 & 24 & $14: 08: 17.10$ & $-03: 46: 17.6$ & 0.37 & $\mathrm{O}$ & $\mathrm{Y}$ & & 8 & 3.9693 & $\mathrm{Y}$ & AG & 6 \\
\hline 050802 & 22 & $14: 37: 05.84$ & $+27: 47: 12.4$ & 0.60 & $\mathrm{O}$ & $\mathrm{Y}$ & & $*$ & 1.7102 & $\mathrm{Y}$ & $\mathrm{AG}$ & 6 \\
\hline 050803 & 22 & $23: 22: 37.86$ & $+05: 47: 08.0$ & 1.40 & $\mathrm{X}$ & $\mathrm{N}$ & $\mathrm{Y}$ & 1 & $4.3_{-2.4}^{+0.6}$ & $\mathrm{~N}$ & host & $*$ \\
\hline 050814 & 20 & $17: 36: 45.39$ & $+46: 20: 21.2$ & 0.30 & $\mathrm{O}$ & $\mathrm{Y}$ & & 9 & 5.3 & $\mathrm{Y}$ & $\mathrm{AG}$ & 10 \\
\hline 050820A & 34 & $22: 29: 38.10$ & $+19: 33: 36.8$ & 0.31 & $\mathrm{O}$ & $\mathrm{Y}$ & & 8 & 2.6147 & $\mathrm{Y}$ & $\mathrm{AG}$ & 6 \\
\hline 050822 & 25 & $03: 24: 27.22$ & $-46: 02: 00.1$ & 0.70 & $\mathrm{X}$ & & & 11 & 1.434 & $\mathrm{~N}$ & host & 12 \\
\hline 050904 & 52 & $00: 54: 50.88$ & $+14: 05: 09.5$ & 0.40 & $\mathrm{O}$ & $\mathrm{Y}$ & & * & 6.295 & $\mathrm{Y}$ & $\mathrm{AG}$ & 13 \\
\hline 050922B & 24 & $00: 23: 13.38$ & $-05: 36: 17.5$ & 1.70 & $\mathrm{X}$ & $\mathrm{N}$ & & 1 & $4.9_{-0.6}^{+0.3}$ & $\mathrm{~N}$ & host & $*$ \\
\hline 050922C & 16 & 21:09:33.08 & $-08: 45: 30.3$ & 0.30 & $\mathrm{O}$ & $\mathrm{Y}$ & & 14 & 2.1995 & $\mathrm{Y}$ & AG & 6 \\
\hline 051001 & 18 & $23: 23: 48.72$ & $-31: 31: 23.6$ & 1.50 & $\mathrm{X}$ & $\mathrm{N}$ & $\mathrm{Y}$ & 11 & 2.4296 & $\mathrm{~N}$ & host & 15 \\
\hline 051006 & 12 & $07: 23: 14.10$ & $+09: 30: 19.4$ & 1.50 & $X$ & $\mathrm{Y}$ & $\mathrm{Y}$ & 1 & 1.059 & $\mathrm{~N}$ & host & 12 \\
\hline 060115 & 17 & 03:36:08.32 & $+17: 20: 42.8$ & 0.31 & $\mathrm{O}$ & $\mathrm{Y}$ & & 8 & 3.5328 & $\mathrm{Y}$ & $\mathrm{AG}$ & 6 \\
\hline 060202 & 22 & $02: 23: 22.94$ & $+38: 23: 03.9$ & 0.50 & I & $\mathrm{N}$ & Y & $*$ & 0.785 & $\mathrm{~N}$ & host & 16 \\
\hline 060204B & 29 & 14:07:14.89 & $+27: 40: 36.2$ & 1.10 & $\mathrm{O}$ & $\mathrm{Y}$ & & 17 & 2.3393 & $\mathrm{~N}$ & host & * \\
\hline 060210 & 76 & 03:50:57.38 & $+27: 01: 34.2$ & 0.60 & $\mathrm{O}$ & $\mathrm{Y}$ & $\mathrm{Y}$ & 18 & 3.9122 & $\mathrm{Y}$ & $\mathrm{AG}$ & 6 \\
\hline 060218 & 65 & $03: 21: 39.68$ & $+16: 52: 01.9$ & 0.28 & $\mathrm{O}$ & $\mathrm{Y}$ & & 8 & 0.0331 & $\mathrm{Y}$ & host & 19 \\
\hline 060306 & 22 & $02: 44: 22.92$ & $-02: 08: 54.1$ & 1.30 & $\mathrm{X}$ & $\mathrm{N}$ & $\mathrm{Y}$ & 11 & 1.559 & $\mathrm{~N}$ & host & 20 \\
\hline $060502 \mathrm{~A}$ & 23 & $16: 03: 42.62$ & $+66: 36: 03.0$ & 0.40 & $\mathrm{O}$ & $\mathrm{Y}$ & & $*$ & 1.5026 & $\mathrm{Y}$ & AG & 6 \\
\hline 060510B & 40 & $15: 56: 29.48$ & $+78: 34: 12.1$ & 0.20 & $\mathrm{O}$ & $\mathrm{Y}$ & & $*$ & 4.9 & $\mathrm{Y}$ & AG & 21 \\
\hline 060522 & 11 & $21: 31: 44.84$ & $+02: 53: 09.7$ & 0.42 & $\mathrm{O}$ & $\mathrm{Y}$ & & 8 & 5.11 & $\mathrm{Y}$ & AG & 22 \\
\hline 060526 & 12 & $15: 31: 18.34$ & $+00: 17: 04.9$ & 0.16 & $\mathrm{O}$ & $\mathrm{Y}$ & & 8 & 3.2213 & $\mathrm{Y}$ & $\mathrm{AG}$ & 6 \\
\hline 060607A & 26 & $21: 58: 50.40$ & $-22: 29: 47.1$ & 0.38 & I & $\mathrm{Y}$ & & 8 & 3.0749 & $\mathrm{Y}$ & AG & 6 \\
\hline 060707 & 16 & $23: 48: 19.06$ & $-17: 54: 17.3$ & 0.37 & $\mathrm{O}$ & $\mathrm{Y}$ & & 8 & 3.4240 & $\mathrm{Y}$ & $\mathrm{AG}$ & 6 \\
\hline 060714 & 29 & $15: 11: 26.41$ & $-06: 33: 58.3$ & 0.43 & $\mathrm{O}$ & $\mathrm{Y}$ & & 8 & 2.7108 & $\mathrm{Y}$ & $\mathrm{AG}$ & 6 \\
\hline 060719 & 15 & 01:13:43.71 & $-48: 22: 51.0$ & 0.29 & $\mathrm{O}$ & $\mathrm{Y}$ & $\mathrm{Y}$ & 8 & 1.5320 & $\mathrm{~N}$ & host & 15 \\
\hline 060729 & 26 & $06: 21: 31.80$ & $-62: 22: 12.3$ & 0.27 & $\mathrm{O}$ & $\mathrm{Y}$ & & 8 & 0.5428 & $\mathrm{Y}$ & $\mathrm{AG}$ & 6 \\
\hline 060814 & 148 & $14: 45: 21.31$ & $+20: 35: 10.5$ & 0.18 & $\mathrm{O}$ & IR & $\mathrm{Y}$ & 8 & 1.9229 & $\mathrm{Y}$ & host & 15 \\
\hline 060908 & 28 & 02:07:18.41 & $+00: 20: 31.3$ & 0.42 & $\mathrm{O}$ & $\mathrm{Y}$ & & 8 & 1.8836 & $\mathrm{Y}$ & $\mathrm{AG}$ & 6 \\
\hline 060912A & 14 & $00: 21: 08.14$ & $+20: 58: 17.4$ & 0.35 & $\mathrm{O}$ & $\mathrm{Y}$ & & 8 & 0.937 & $\mathrm{Y}$ & host & 23 \\
\hline 060927 & 11 & 21:58:12.01 & $+05: 21: 48.6$ & 0.19 & $\mathrm{O}$ & $\mathrm{Y}$ & & 8 & 5.467 & $\mathrm{Y}$ & AG & 24 \\
\hline 061007 & 450 & 03:05:19.58 & $-50: 30: 02.3$ & 0.33 & $\mathrm{O}$ & $\mathrm{Y}$ & & 8 & 1.2622 & $\mathrm{Y}$ & $\mathrm{AG}$ & 6 \\
\hline 061021 & 30 & 09:40:36.12 & $-21: 57: 04.8$ & 0.30 & $\mathrm{O}$ & $\mathrm{Y}$ & & 8 & 0.3463 & $\mathrm{~N}$ & $\mathrm{AG}$ & 6 \\
\hline 061110A & 11 & $22: 25: 09.84$ & $-02: 15: 31.4$ & 0.37 & $\mathrm{O}$ & $\mathrm{Y}$ & & 8 & 0.7578 & $\mathrm{Y}$ & $\mathrm{AG}$ & 6 \\
\hline 061110B & 14 & $21: 35: 40.39$ & $+06: 52: 34.0$ & 0.24 & $\mathrm{O}$ & $\mathrm{Y}$ & & 8 & 3.4344 & $\mathrm{Y}$ & $\mathrm{AG}$ & 6 \\
\hline 061121 & 139 & 09:48:54.55 & $-13: 11: 42.9$ & 0.36 & $\mathrm{O}$ & $\mathrm{Y}$ & & 8 & 1.3145 & $\mathrm{Y}$ & $\mathrm{AG}$ & 6 \\
\hline 061202 & 35 & 07:02:06.09 & $-74: 41: 54.7$ & 1.40 & $\mathrm{X}$ & & & 1 & 2.253 & $\mathrm{~N}$ & host & $*$ \\
\hline 061222A & 81 & 23:53:03.41 & $+46: 31: 58.6$ & 0.30 & I & IR & $\mathrm{Y}$ & 25 & 2.088 & $\mathrm{~N}$ & host & 26 \\
\hline 070110 & 16 & 00:03:39.27 & $-52: 58: 27.2$ & 0.30 & $\mathrm{O}$ & $\mathrm{Y}$ & & 8 & 2.3521 & $\mathrm{Y}$ & $\mathrm{AG}$ & 6 \\
\hline 070129 & 30 & 02:28:00.94 & $+11: 41: 04.1$ & 0.31 & $\mathrm{O}$ & $\mathrm{Y}$ & & 8 & 2.3384 & $\mathrm{~N}$ & host & 15 \\
\hline 070223 & 19 & $10: 13: 48.39$ & $+43: 08: 00.7$ & 0.40 & I & $\mathrm{Y}$ & & 27 & 1.6295 & $\mathrm{~N}$ & host & * \\
\hline 070306 & 55 & $09: 52: 23.30$ & $+10: 28: 55.2$ & 0.31 & $\mathrm{O}$ & IR & $\mathrm{Y}$ & 8 & 1.4959 & $\mathrm{Y}$ & host & 28 \\
\hline 070318 & 26 & $03: 13: 56.81$ & $-42: 56: 46.1$ & 0.35 & $\mathrm{O}$ & $\mathrm{Y}$ & & 8 & 0.840 & $\mathrm{Y}$ & $\mathrm{AG}$ & 29 \\
\hline 070328 & 91 & $04: 20: 27.73$ & $-34: 04: 00.5$ & 1.40 & $\mathrm{X}$ & & & 1 & 2.0627 & $\mathrm{~N}$ & host & 20 \\
\hline 070419B & 75 & $21: 02: 49.77$ & $-31: 15: 49.0$ & 0.39 & $\mathrm{O}$ & $\mathrm{Y}$ & & 8 & 1.9588 & $\mathrm{~N}$ & host & 15 \\
\hline 070508 & 201 & $20: 51: 11.70$ & $-78: 23: 05.1$ & 0.40 & $\mathrm{O}$ & $\mathrm{Y}$ & $\mathrm{Y}$ & $*$ & 0.82 & $\mathrm{Y}$ & host & 30 \\
\hline 070521 & 81 & $16: 10: 38.61$ & $+30: 15: 21.9$ & 1.40 & $X$ & $\mathrm{~N}$ & $\mathrm{Y}$ & 1 & 2.0865 & $\mathrm{~N}$ & host & 20 \\
\hline 070621 & 44 & 21:35:10.09 & $-24: 49: 03.1$ & 1.40 & $X$ & $\mathrm{~N}$ & $\mathrm{Y}$ & 1 & $<5.5$ & $\mathrm{~N}$ & host & $*$ \\
\hline 070721B & 36 & $02: 12: 32.96$ & $-02: 11: 40.8$ & 0.38 & $\mathrm{O}$ & $\mathrm{Y}$ & & 8 & 3.6298 & $\mathrm{Y}$ & $\mathrm{AG}$ & 6 \\
\hline 070808 & 13 & $00: 27: 03.36$ & $+01: 10: 33.9$ & 1.50 & $\mathrm{X}$ & $\mathrm{N}$ & $\mathrm{Y}$ & 1 & $1.35 \pm 0.85$ & $\mathrm{~N}$ & $N_{\mathrm{H}, \mathrm{X}}$ & $*$ \\
\hline
\end{tabular}


Table 3

(Continued)

\begin{tabular}{|c|c|c|c|c|c|c|c|c|c|c|c|c|}
\hline GRB & $S_{\mathrm{BAT}}{ }^{\mathrm{a}}$ & R. A. ${ }^{\mathrm{b}}$ & Decl. $^{\text {b }}$ & Unc. $^{c}$ & Type. $^{\mathrm{d}}$ & OT? ${ }^{\mathrm{e}}$ & Dusty? ${ }^{\mathrm{f}}$ & References $^{\mathrm{i}}$ & $z$ & Early? & Origin $^{\mathrm{h}}$ & References \\
\hline 071020 & 23 & 07:58:39.78 & $+32: 51: 40.4$ & 0.35 & I & $\mathrm{Y}$ & & 31 & 2.1462 & $\mathrm{Y}$ & $\mathrm{AG}$ & 6 \\
\hline 071021 & 14 & $22: 42: 34.30$ & $+23: 43: 06.2$ & 0.60 & $\mathrm{O}$ & IR & $\mathrm{Y}$ & 32 & 2.4520 & $\mathrm{Y}$ & host & 15 \\
\hline 071025 & 73 & $23: 40: 17.07$ & $+31: 46: 42.8$ & 0.35 & I & $\mathrm{Y}$ & $\mathrm{Y}$ & 33 & $4.8_{-0.4}^{+0.4}$ & $\mathrm{~N}$ & $\mathrm{AG}$ & 34 \\
\hline $071112 \mathrm{C}$ & 30 & 02:36:50.95 & $+28: 22: 16.8$ & 0.41 & $\mathrm{O}$ & $\mathrm{Y}$ & & 5 & 0.8227 & $\mathrm{Y}$ & $\mathrm{AG}$ & 6 \\
\hline 080205 & 20 & 06:33:00.63 & $+62: 47: 31.7$ & 0.50 & $\mathrm{U}$ & $\mathrm{Y}$ & & 35 & $2.72_{-0.74}^{+0.24}$ & $\mathrm{~N}$ & host & * \\
\hline 080207 & 61 & 13:50:02.97 & $+07: 30: 07.3$ & 0.50 & $\mathrm{X}$ & $\mathrm{N}$ & $\mathrm{Y}$ & 36 & 2.0858 & $\mathrm{Y}$ & host & 15 \\
\hline 080210 & 18 & $16: 45: 04.01$ & $+13: 49: 35.6$ & 0.60 & $\mathrm{U}$ & $\mathrm{Y}$ & & 35 & 2.6419 & $\mathrm{Y}$ & $\mathrm{AG}$ & 6 \\
\hline 080310 & 23 & $14: 40: 13.80$ & $-00: 10: 30.7$ & 0.40 & $\mathrm{O}$ & $\mathrm{Y}$ & & 37 & 2.4274 & $\mathrm{Y}$ & $\mathrm{AG}$ & 6 \\
\hline 080319A & 44 & $13: 45: 20.01$ & $+44: 04: 48.4$ & 0.70 & $\mathrm{O}$ & $\mathrm{Y}$ & & 38 & 2.0265 & $\mathrm{~N}$ & host & $*$ \\
\hline 080319B & 850 & $14: 31: 40.99$ & $+36: 18: 08.7$ & 0.30 & $\mathrm{O}$ & $\mathrm{Y}$ & & * & 0.9382 & $\mathrm{Y}$ & $\mathrm{AG}$ & 6 \\
\hline 080325 & 49 & $18: 31: 34.23$ & $+36: 31: 24.8$ & 0.30 & I & IR & Y & 39 & 1.78 & $\mathrm{~N}$ & host & 40 \\
\hline 080411 & 265 & $02: 31: 55.21$ & $-71: 18: 07.3$ & 0.50 & $\mathrm{U}$ & $\mathrm{Y}$ & & 35 & 1.0301 & $\mathrm{Y}$ & AG & 6 \\
\hline 080413A & 35 & 19:09:11.75 & $-27: 40: 40.4$ & 0.40 & $\mathrm{O}$ & $\mathrm{Y}$ & & $*$ & 2.4330 & $\mathrm{Y}$ & AG & 6 \\
\hline 080413B & 33 & $21: 44: 34.66$ & $-19: 58: 52.4$ & 0.50 & $\mathrm{U}$ & $\mathrm{Y}$ & & 35 & 1.1014 & $\mathrm{Y}$ & $\mathrm{AG}$ & 6 \\
\hline 080430 & 12 & $11: 01: 14.76$ & $+51: 41: 08.0$ & 0.40 & $\mathrm{O}$ & $\mathrm{Y}$ & & 41 & 0.767 & $\mathrm{Y}$ & AG & 42 \\
\hline 080603B & 25 & $11: 46: 07.67$ & $+68: 03: 39.8$ & 0.30 & $\mathrm{U}$ & $\mathrm{Y}$ & & 35 & 2.6892 & $\mathrm{Y}$ & $\mathrm{AG}$ & 6 \\
\hline 080605 & 134 & $17: 28: 30.04$ & $+04: 00: 56.0$ & 0.30 & $\mathrm{O}$ & $\mathrm{Y}$ & $\mathrm{Y}$ & $*$ & 1.6403 & $\mathrm{Y}$ & AG & 6 \\
\hline 080607 & 247 & $12: 59: 47.21$ & $+15: 55: 10.5$ & 0.40 & $\mathrm{O}$ & $\mathrm{Y}$ & $\mathrm{Y}$ & * & 3.0368 & $\mathrm{Y}$ & $\mathrm{AG}$ & 6 \\
\hline 080710 & 14 & 00:33:05.63 & $+19: 30: 05.4$ & 0.30 & $\mathrm{O}$ & Y & & * & 0.8454 & $\mathrm{Y}$ & AG & 6 \\
\hline 080721 & 141 & $14: 57: 55.84$ & $-11: 43: 24.6$ & 0.40 & $\mathrm{O}$ & Y & & 5 & 2.5914 & $\mathrm{Y}$ & $\mathrm{AG}$ & 6 \\
\hline 080804 & 38 & 21:54:40.18 & $-53: 11: 04.8$ & 0.35 & $\mathrm{O}$ & $\mathrm{Y}$ & & $*$ & 2.2045 & $\mathrm{Y}$ & AG & 6 \\
\hline 080805 & 27 & $20: 56: 53.45$ & $-62: 26: 40.0$ & 0.60 & $\mathrm{O}$ & $\mathrm{Y}$ & & 43 & 1.5042 & $\mathrm{Y}$ & $\mathrm{AG}$ & 6 \\
\hline 080810 & 46 & $23: 47: 10.49$ & $+00: 19: 11.3$ & 0.30 & $\mathrm{O}$ & $\mathrm{Y}$ & & $*$ & 3.3604 & $\mathrm{Y}$ & $\mathrm{AG}$ & 6 \\
\hline 080916A & 42 & $22: 25: 06.23$ & $-57: 01: 22.9$ & 0.40 & $\mathrm{O}$ & $\mathrm{Y}$ & & * & 0.6887 & $\mathrm{Y}$ & $\mathrm{AG}$ & 6 \\
\hline 080928 & 24 & $06: 20: 16.83$ & $-55: 11: 58.7$ & 0.40 & $\mathrm{O}$ & $\mathrm{Y}$ & & $*$ & 1.6919 & $\mathrm{Y}$ & AG & 6 \\
\hline 081008 & 43 & $18: 39: 49.86$ & $-57: 25: 53.0$ & 0.60 & $\mathrm{U}$ & $\mathrm{Y}$ & & 35 & 1.967 & $\mathrm{Y}$ & $\mathrm{AG}$ & 44 \\
\hline 081029 & 21 & 23:07:05.35 & $-68: 09: 19.7$ & 0.50 & $\mathrm{U}$ & $\mathrm{Y}$ & & 35 & 3.8479 & $\mathrm{Y}$ & AG & 45 \\
\hline 081109A & 40 & 22:03:09.61 & $-54: 42: 40.1$ & 0.35 & $\mathrm{O}$ & Y & Y & 46 & 0.9787 & $\mathrm{~N}$ & host & 15 \\
\hline 081118 & 12 & $05: 30: 22.18$ & $-43: 18: 05.1$ & 0.60 & $\mathrm{O}$ & $\mathrm{Y}$ & & 47 & 2.58 & $\mathrm{Y}$ & $\mathrm{AG}$ & 48 \\
\hline 081121 & 42 & 05:57:06.17 & $-60: 36: 09.8$ & 0.60 & $\mathrm{U}$ & $\mathrm{Y}$ & & 35 & 2.512 & $\mathrm{Y}$ & $A G$ & 49 \\
\hline 081128 & 23 & $01: 23: 13.10$ & $+38: 07: 38.7$ & 0.20 & $\mathrm{O}$ & $\mathrm{Y}$ & & $*$ & $<3.4$ & $\mathrm{~N}$ & host & $*$ \\
\hline 081210 & 19 & $04: 41: 56.20$ & $-11: 15: 26.8$ & 0.64 & $\mathrm{U}$ & $\mathrm{Y}$ & & 35 & 2.0631 & $\mathrm{~N}$ & host & 20 \\
\hline 081221 & 189 & 01:03:10.16 & $-24: 32: 51.6$ & 0.25 & I & IR & $\mathrm{Y}$ & $*$ & 2.26 & $\mathrm{~N}$ & host & 50 \\
\hline 081222 & 52 & $01: 30: 57.60$ & $-34: 05: 41.6$ & 0.50 & $\mathrm{U}$ & $\mathrm{Y}$ & & 35 & 2.77 & $\mathrm{Y}$ & AG & 51 \\
\hline 090313 & 15 & $13: 13: 36.20$ & $+08: 05: 49.6$ & 0.70 & $\mathrm{O}$ & $\mathrm{Y}$ & & 52 & 3.375 & $\mathrm{Y}$ & $\mathrm{AG}$ & 53 \\
\hline 090404 & 31 & $15: 56: 57.52$ & $+35: 30: 57.5$ & 0.40 & $\mathrm{R}$ & $\mathrm{N}$ & $\mathrm{Y}$ & 54 & $3.0_{-1.8}^{+0.8}$ & $\mathrm{~N}$ & host & 16 \\
\hline 090417B & 23 & $13: 58: 46.58$ & $+47: 01: 04.8$ & 1.00 & $\mathrm{X}$ & $\mathrm{N}$ & Y & 11 & 0.345 & $\mathrm{~N}$ & host & 55 \\
\hline 090418A & 47 & $17: 57: 15.16$ & $+33: 24: 20.9$ & 0.40 & $\mathrm{O}$ & $\mathrm{Y}$ & & 56 & 1.608 & $\mathrm{Y}$ & $\mathrm{AG}$ & 57 \\
\hline 090424 & 218 & $12: 38: 05.08$ & $+16: 50: 15.0$ & 0.60 & $\mathrm{O}$ & $\mathrm{Y}$ & & * & 0.544 & $\mathrm{Y}$ & host & 58 \\
\hline 090516A & 90 & 09:13:02.60 & $-11: 51: 15.0$ & 0.40 & $\mathrm{O}$ & $\mathrm{Y}$ & & $*$ & 4.109 & Y & AG & 59 \\
\hline 090519 & 12 & 09:29:07.00 & $+00: 10: 48.9$ & 0.60 & $\mathrm{O}$ & $\mathrm{Y}$ & & 60 & 3.85 & $\mathrm{Y}$ & AG & 61 \\
\hline 090530 & 11 & $11: 57: 40.49$ & $+26: 35: 37.7$ & 0.40 & $\mathrm{O}$ & $\mathrm{Y}$ & & * & 1.266 & $\mathrm{~N}$ & host & 62 \\
\hline 090618 & 1090 & $19: 35: 58.73$ & $+78: 21: 24.3$ & 0.50 & $\mathrm{O}$ & $\mathrm{Y}$ & & * & 0.54 & $\mathrm{Y}$ & AG & 63 \\
\hline 090709A & 253 & $19: 19: 42.64$ & $+60: 43: 39.3$ & 0.50 & I & $\mathrm{Y}$ & $\mathrm{Y}$ & 64 & $1.8_{-0.7}^{+0.5}$ & $\mathrm{~N}$ & host & 16 \\
\hline 090715B & 57 & $16: 45: 21.63$ & $+44: 50: 21.0$ & 0.40 & $\mathrm{O}$ & $\mathrm{Y}$ & & $*$ & 3.00 & $\mathrm{Y}$ & $\mathrm{AG}$ & 65 \\
\hline 090812 & 57 & $23: 32: 48.56$ & $-10: 36: 17.2$ & 0.40 & $\mathrm{O}$ & Y & & 66 & 2.452 & $\mathrm{Y}$ & AG & 67 \\
\hline 090814A & 13 & $15: 58: 26.39$ & $+25: 37: 52.4$ & 0.40 & $\mathrm{O}$ & $\mathrm{Y}$ & & * & 0.696 & $\mathrm{Y}$ & AG & 68 \\
\hline 090926B & 71 & 03:05:13.93 & $-39: 00: 22.2$ & 1.40 & $\mathrm{X}$ & $\mathrm{N}$ & Y & 1 & 1.24 & $\mathrm{Y}$ & AG & 69 \\
\hline 091018 & 14 & 02:08:44.63 & $-57: 32: 53.8$ & 0.50 & $\mathrm{O}$ & $\mathrm{Y}$ & & $*$ & 0.971 & $\mathrm{Y}$ & AG & 70 \\
\hline 091029 & 24 & $04: 00: 42.62$ & $-55: 57: 20.0$ & 0.50 & $\mathrm{O}$ & $\mathrm{Y}$ & & * & 2.752 & $\mathrm{Y}$ & AG & 71 \\
\hline 091109A & 16 & $20: 37: 01.82$ & $-44: 09: 29.6$ & 0.40 & $\mathrm{O}$ & $\mathrm{Y}$ & & * & 3.076 & $\mathrm{Y}$ & AG & 72 \\
\hline 091127 & 84 & $02: 26: 19.89$ & $-18: 57: 08.5$ & 0.55 & $\mathrm{U}$ & $\mathrm{Y}$ & & 35 & 0.490 & $\mathrm{Y}$ & host & 73 \\
\hline 091208B & 32 & 01:57:34.10 & $+16: 53: 22.6$ & 0.25 & $\mathrm{O}$ & $\mathrm{Y}$ & & $*$ & 1.0633 & $\mathrm{Y}$ & $\mathrm{AG}$ & 74 \\
\hline $100305 \mathrm{~A}$ & 15 & $11: 13: 28.07$ & $+42: 24: 14.3$ & 1.00 & $\mathrm{X}$ & $\mathrm{N}$ & $\mathrm{Y}$ & 11 & & $\mathrm{~N}$ & & \\
\hline $100615 \mathrm{~A}$ & 50 & $11: 48: 49.34$ & $-19: 28: 52.0$ & 0.70 & $\mathrm{X}$ & $\mathrm{N}$ & Y & 75 & 1.398 & $\mathrm{~N}$ & host & 76 \\
\hline
\end{tabular}


Table 3

(Continued)

\begin{tabular}{|c|c|c|c|c|c|c|c|c|c|c|c|c|}
\hline GRB & $S_{\mathrm{BAT}^{\mathrm{a}}}^{\mathrm{a}}$ & R. A. ${ }^{b}$ & Decl. $^{\mathrm{b}}$ & Unc. $^{\mathrm{c}}$ & Type. $^{d}$ & OT? ${ }^{\mathrm{e}}$ & Dusty? ${ }^{\mathrm{f}}$ & References $^{\mathrm{i}}$ & $z$ & Early $^{\mathrm{g}}$ & Origin $^{h}$ & References $^{\mathrm{i}}$ \\
\hline $100621 \mathrm{~A}$ & 210 & 21:01:13.08 & $-51: 06: 22.5$ & 0.40 & I & $\mathrm{Y}$ & Y & 77 & 0.542 & Y & host & 78 \\
\hline 100728B & 17 & $02: 56: 13.46$ & $+00: 16: 52.1$ & 0.52 & $\mathrm{U}$ & Y & & 35 & 2.106 & Y & $\mathrm{AG}$ & 79 \\
\hline $100802 \mathrm{~A}$ & 36 & 00:09:52.38 & $+47: 45: 18.8$ & 0.50 & $\mathrm{O}$ & Y & & 80 & $<3.1$ & $\mathrm{~N}$ & host & * \\
\hline $100814 \mathrm{~A}$ & 90 & 01:29:53.59 & $-17: 59: 43.5$ & 0.40 & $\mathrm{O}$ & Y & & * & 1.44 & Y & $\mathrm{AG}$ & 81 \\
\hline $110205 \mathrm{~A}$ & 170 & $10: 58: 31.10$ & $+67: 31: 30.5$ & 0.30 & $\mathrm{O}$ & Y & & 82 & 2.22 & Y & $\mathrm{AG}$ & 83 \\
\hline 110709B & 94 & $10: 58: 37.11$ & $-23: 27: 16.7$ & 0.70 & $\mathrm{R}$ & $\mathrm{N}$ & $\mathrm{Y}$ & 84 & $2.09 ?$ & $\mathrm{~N}$ & host & $*$ \\
\hline $120119 A$ & 170 & 08:00:06.94 & $-09: 04: 53.8$ & 0.30 & $\mathrm{O}$ & Y & Y & 85 & 1.728 & Y & $\mathrm{AG}$ & 85 \\
\hline $120308 \mathrm{~A}$ & 12 & $14: 36: 20.05$ & $+79: 41: 12.2$ & 0.55 & U & $\mathrm{Y}$ & & 35 & $<3.7$ & $\mathrm{~N}$ & host & * \\
\hline
\end{tabular}

Notes.

${ }^{\text {a }}$ Swift-BAT prompt-emission fluence (15-150 keV) in units of $10^{-7} \mathrm{erg} \mathrm{cm}^{-2}$. From Sakamoto et al. (2011) if available, otherwise from the Swift GRB table.

${ }^{\mathrm{b}}$ Best afterglow position (J2000), relative to the 2MASS astrometric system.

${ }^{\mathrm{c}}$ Position uncertainty $(\operatorname{arcsec})$, including an estimate of the systematic uncertainty.

${ }^{\mathrm{d}}$ First letter of the wavelength at which the position was reported: X-ray, UV, optical, IR, or radio/millimeter.

e Whether or not a variable optical afterglow was reported. "IR" indicates that an NIR afterglow was reported but not an optical $(\lambda<1 \mu \mathrm{m})$ afterglow. Blank if no observations were conducted or reported limits are very shallow.

${ }^{\mathrm{f}}$ Whether the afterglow shows evidence of being dust-obscured and/or "dark," based on $\beta_{\mathrm{OX}}$ or the optical-NIR color. From a variety of sources, including Perley et al. (2013), Zafar et al. (2012), and Greiner et al. (2011).

${ }^{\mathrm{g}}$ Whether or not a redshift estimate was promptly publicly available. (If "N," the redshift was measured by late-time observations of the host galaxy or only a limit could be provided.)

${ }^{\mathrm{h}}$ Source of the redshift measurement/limit: observations of the optical afterglow (AG), of the host galaxy (host), or of the X-ray afterglow ( $N_{\mathrm{H}, \mathrm{X}}$ ).

${ }^{\mathrm{i}}$ References for the afterglow position (middle column) and redshift (right column), given below.

Sources as follows-*: This work, (1): Evans et al. 2009; (2): D. Malesani 2015, private communication; (3): Berger et al. 2005; (4): Mulchaey \& Berger 2005; (5): Y. Urata 2015, private communication; (6): Fynbo et al. 2009b; (7): Foley et al. 2005; (8): D. Malesani et al. 2015, in preparation; (9): Cenko 2005; (10): Jakobsson et al. 2006; (11): Butler \& Kocevski 2007; (12): Hjorth et al. 2012; (13): Kawai et al. 2006; (14): Jakobsson et al. 2005; (15): Krühler et al. 2012; (16): Perley et al. 2013; (17): Guidorzi et al. 2006; (18): Mundell et al. 2006; (19): Mirabal \& Halpern 2006; (20): Krühler et al. 2015; (21): Price 2006; (22): Cenko et al. 2006a; (23): Levan et al. 2007; (24): Ruiz-Velasco et al. 2007; (25): Cenko \& Fox 2006; (26): Perley et al. 2009c; (27): Rol et al. 2007; (28): Jaunsen et al. 2008; (29): Chen et al. 2007; (30): Jakobsson et al. 2007; (31): Bloom et al. 2007; (32): Castro-Tirado et al. 2007; (33): Bloom 2007; (34): Perley et al. 2010; (35): Swift GRB Table (36): Svensson et al. 2012; (37): Chornock et al. 2008; (38): Cenko 2008; (39): Hashimoto et al. 2010; (40): Hashimoto et al. 2015; (41): Rinner \& Kugel 2008; (42): Cucchiara \& Fox 2008; (43): Krühler et al. 2008; (44): Cucchiara et al. 2008a; (45): D’Elia et al. 2008a; (46): T. Krühler 2015, private communication; (47): D’Avanzo et al. 2008; (48): D’Elia et al. 2008b; (49): Berger \& Rauch 2008; (50): Salvaterra et al. 2012; (51): Cucchiara et al. 2008b; (52): Chornock et al. 2009b; (53): Chornock et al. 2009d; (54): Castro-Tirado et al. 2009; (55): Berger \& Fox 2009; (56): Pavlenko et al. 2009; (57): Chornock et al. 2009a; (58): Chornock et al. 2009c; (59): de Ugarte Postigo et al. 2009b; (60): Thöne et al. 2009a; (61): Thöne et al. 2009b; (62): Goldoni et al. 2013; (63): Cenko et al. 2009a; (64): Morgan et al. 2009; (65): Wiersema et al. 2009; (66): Cenko 2009; (67): de Ugarte Postigo et al. 2009a; (68): Jakobsson et al. 2009; (69): Fynbo et al. 2009a; (70): Chen et al. 2009; (71): Chornock et al. 2009e; (72): Rau et al. 2010; (73): Cucchiara et al. 2009; (74): Perley et al. 2009a; (75): Butler et al. 2010b; (76): Krühler et al. 2013; (77): Updike et al. 2010; (78): Milvang-Jensen et al. 2010; (79): Flores et al. 2010; (80): de Ugarte Postigo et al. 2009d; (81): O’Meara et al. 2010; (82): Mundell et al. 2011; (83): Cenko et al. 2011; (84): Zauderer \& Berger 2011; (85): Morgan et al. 2014.

potentially very luminous galaxy can appear quite faint in these bands if it is heavily dust obscured.)

Observations of many targets in our sample were already present in the Spitzer Legacy Archive; the remaining targets were observed as part of our Cycle 9 Large Program at $3.6 \mu \mathrm{m}$ with an exposure time chosen depending on the redshift (typically between 0.5 and $5 \mathrm{hr}$; see Paper II for details). Observations at $4.5 \mu \mathrm{m}$ were also acquired for some targets, in particular, for those at unknown redshift and for GRBs designated as "dark," in order to provide better photometric redshift estimates and to model dust extinction in high-redshift galaxies.

\subsection{Optical and Near-infrared Observations}

Spitzer observations alone provide an estimate of a galaxy's total stellar mass, but they do not constrain the other properties of a galaxy, including the nature of the young stellar population that (presumably) produced the GRB. In addition, because of Spitzer's large PSF size $\left(\sim 2^{\prime \prime}\right)$, it is not straightforward to uniquely identify the host galaxy based on Spitzer observations alone, even in possession of a precise afterglow localization: deep observations approach the confusion limit, and it is not always clear whether an extended source at the afterglow location represents an extended host galaxy or a blend of the host galaxy and a foreground object.

For both these reasons, we have obtained a large volume of imaging at optical and NIR wavelengths of all of our targets. Given the wide range in redshifts and luminosities of the galaxies targeted by our survey and its all-sky nature (requiring different observational facilities to cover the northern and southern regions, and observing runs scattered throughout the year), this follow-up is necessarily heterogeneous and usually tailored to each individual target. Typically, we try to obtain at least one deep $\left(R_{\text {lim }} \sim 26\right)$ ground-based optical (rest-frame $\mathrm{UV}$ at $z>1$ ) measurement and then obtain additional filters to the extent possible given the brightness of the target and the resources available; we also employ archival observations from a variety of previous surveys and from the Gemini and VLT archives.

Most observations were conducted using the Low Resolution Imaging Spectrometer (LRIS; Oke et al. 1995) on Keck I, with substantial imaging also coming from the Gemini Multi-Object Spectographs (GMOS; Hook et al. 2004) at Gemini-North and Gemini-South, the Optical System for Imaging and lowIntermediate-Resolution Integrated Spectroscopy (OSIRIS) at 
Table 4

Host Galaxy Photometry Used in Photometric Redshifts and Upper Limits

\begin{tabular}{|c|c|c|c|c|}
\hline GRB & Filter & $\mathrm{mag}^{\mathrm{a}}$ & Unc. & Instrument \\
\hline 050128 & $R$ & 24.84 & 0.10 & VLT/FORS1 \\
\hline \multirow[t]{6}{*}{050803} & $g$ & $>27.74$ & $\cdots$ & GTC/OSIRIS \\
\hline & $R$ & 26.32 & 0.22 & VLT/FORS2 \\
\hline & $i$ & 26.45 & 0.50 & GTC/OSIRIS \\
\hline & F160W & 25.79 & 0.18 & $H S T / \mathrm{WFC} 3-\mathrm{IR}$ \\
\hline & 3.6 & 25.49 & 0.42 & Spitzer/IRAC \\
\hline & 4.5 & $>25.00$ & $\ldots$ & Spitzer/IRAC \\
\hline \multirow[t]{7}{*}{ 050922B } & $g$ & 27.63 & 0.50 & Keck/LRIS \\
\hline & $R$ & 26.44 & 0.22 & Keck/LRIS \\
\hline & $i$ & 25.25 & 0.14 & GTC/OSIRIS \\
\hline & $z$ & 25.09 & 0.34 & GTC/OSIRIS \\
\hline & Ks & $>22.17$ & $\ldots$ & VLT/ISAAC \\
\hline & 3.6 & 24.59 & 0.22 & Spitzer/IRAC \\
\hline & 4.5 & 24.80 & 0.42 & Spitzer/IRAC \\
\hline \multirow[t]{11}{*}{ 060204B } & $u$ & 25.33 & 0.15 & Keck/LRIS \\
\hline & $B$ & 24.93 & 0.06 & Keck/LRIS \\
\hline & $g$ & 24.49 & 0.03 & Keck/LRIS \\
\hline & $R$ & 24.12 & 0.07 & Keck/LRIS \\
\hline & $i$ & 23.86 & 0.07 & Keck/LRIS \\
\hline & $z$ & 24.20 & 0.18 & Keck/LRIS \\
\hline & $Y$ & 23.22 & 0.16 & Keck/MOSFIRE \\
\hline & $J$ & 22.46 & 0.40 & Keck/MOSFIRE \\
\hline & Ks & 20.35 & 0.40 & Keck/MOSFIRE \\
\hline & 3.6 & 22.74 & 0.05 & Spitzer/IRAC \\
\hline & 4.5 & 22.00 & 0.30 & Spitzer/IRAC \\
\hline \multirow[t]{5}{*}{070808} & $g$ & $>27.50$ & $\ldots$ & Keck/LRIS \\
\hline & $R$ & 26.71 & 0.33 & VLT/FORS2 \\
\hline & Ks & 21.77 & 0.37 & VLT/ISAAC \\
\hline & 3.6 & 23.57 & 0.10 & Spitzer/IRAC \\
\hline & 4.5 & 23.80 & 0.20 & Spitzer/IRAC \\
\hline \multirow[t]{12}{*}{ 080319A } & $u$ & 25.30 & 0.13 & Keck/LRIS \\
\hline & $B$ & 25.02 & 0.10 & Keck/LRIS \\
\hline & $g$ & 24.50 & 0.04 & Keck/LRIS \\
\hline & $R$ & 24.06 & 0.12 & Keck/LRIS \\
\hline & $i$ & 24.24 & 0.08 & Keck/LRIS \\
\hline & $z$ & 24.26 & 0.12 & Keck/LRIS \\
\hline & $Y$ & 23.61 & 0.17 & Keck/MOSFIRE \\
\hline & $J$ & 22.60 & 0.12 & Keck/MOSFIRE \\
\hline & $H$ & 21.64 & 0.12 & Keck/MOSFIRE \\
\hline & Ks & 21.05 & 0.13 & Keck/MOSFIRE \\
\hline & 3.6 & 22.63 & 0.03 & Spitzer/IRAC \\
\hline & 4.5 & 22.60 & 0.04 & Spitzer/IRAC \\
\hline \multirow[t]{6}{*}{081128} & $B$ & 26.14 & 0.24 & Keck/LRIS \\
\hline & $g$ & 25.80 & 0.15 & Keck/LRIS \\
\hline & V & 25.37 & 0.13 & Keck/LRIS \\
\hline & $i$ & 25.24 & 0.19 & Keck/LRIS \\
\hline & $z$ & 24.97 & 0.33 & Keck/LRIS \\
\hline & 3.6 & 25.20 & 0.31 & Spitzer/IRAC \\
\hline \multirow[t]{4}{*}{$100802 \mathrm{~A}$} & $B$ & 26.44 & 0.16 & Keck/LRIS \\
\hline & $R$ & 25.23 & 0.25 & Keck/LRIS \\
\hline & $i$ & 26.23 & 0.32 & Keck/LRIS \\
\hline & 3.6 & 25.47 & 0.43 & Spitzer/IRAC \\
\hline \multirow[t]{5}{*}{$120308 \mathrm{~A}$} & $B$ & $>26.67$ & $\cdots$ & Keck/LRIS \\
\hline & $g$ & 26.33 & 0.17 & Keck/LRIS \\
\hline & $R$ & 25.77 & 0.22 & Keck/LRIS \\
\hline & $I$ & 24.46 & 0.23 & Keck/LRIS \\
\hline & 3.6 & 24.98 & 0.27 & Spitzer/IRAC \\
\hline
\end{tabular}

Note.

${ }^{\text {a }}$ In the Sloan Digital Sky Survey (SDSS; for ugriz), Vega (BRIY), or 2MASS (JHKs) magnitude systems; AB magnitudes are used for the IRAC (3.6 and 4.5 $\mu \mathrm{m})$ filters. Measurements are uncorrected for foreground extinction.
Table 5

GRB Rate Measurements

\begin{tabular}{|c|c|c|c|c|c|c|c|}
\hline \multirow[b]{2}{*}{$z_{\min }$} & \multirow[b]{2}{*}{$z_{\max }$} & \multirow[b]{2}{*}{$E_{\text {iso,sens }}{ }^{a}$} & \multirow[b]{2}{*}{$C_{\text {complete }}{ }^{\mathrm{b}}$} & \multicolumn{2}{|c|}{ SHOALS } & \multicolumn{2}{|c|}{ All Swift } \\
\hline & & & & $N^{\mathrm{c}}$ & $\rho_{\mathrm{GRB}, 51}{ }^{\mathrm{d}}$ & $N^{\mathrm{c}}$ & $\rho_{\mathrm{GRB}, 51}{ }^{\mathrm{d}}$ \\
\hline 0.10 & 0.50 & 0.90 & 0.91 & 2 & $0.21_{-0.09}^{+0.33}$ & 5 & $0.26_{-0.10}^{+0.21}$ \\
\hline 0.50 & 1.00 & 3.19 & 2.83 & 13 & $1.47_{-0.39}^{+0.59}$ & 26 & $1.46_{-0.29}^{+0.39}$ \\
\hline 1.00 & 1.50 & 6.22 & 5.17 & 11 & $1.80_{-0.51}^{+0.81}$ & 28 & $2.28_{-0.44}^{+0.58}$ \\
\hline 1.50 & 2.00 & 9.60 & 7.65 & 16 & $3.95_{-0.96}^{+1.36}$ & 31 & $3.81_{-0.71}^{+0.91}$ \\
\hline 2.00 & 2.65 & 14.19 & 10.8 & 27 & $8.34_{-1.58}^{+1.91}$ & 45 & $6.92_{-1.06}^{+1.26}$ \\
\hline 2.65 & 3.50 & 20.23 & 14.9 & 17 & $6.90_{-1.64}^{+2.27}$ & 38 & $7.67_{-1.28}^{+1.58}$ \\
\hline 3.50 & 4.50 & 27.19 & 19.5 & 7 & $4.17_{-1.39}^{+2.61}$ & 15 & $4.44_{-1.13}^{+1.73}$ \\
\hline 4.50 & 6.00 & 37.15 & 25.8 & 5 & $3.75_{-1.39}^{+2.98}$ & 9 & $3.36_{-1.03}^{+1.85}$ \\
\hline 6.00 & 8.00 & 49.54 & 33.5 & 1 & $1.12_{-0.53}^{+3.14}$ & 2 & $1.12_{-0.51}^{+1.81}$ \\
\hline 8.00 & 10.00 & 61.03 & 40.4 & 0 & $0.00_{-0.00}^{+3.27}$ & 0 & $0.00_{-0.00}^{+1.65}$ \\
\hline
\end{tabular}

Notes.

${ }^{a} E_{\text {iso }}$ sensitivity threshold for inclusion of a GRB in the count, in units of $10^{51}$ erg as measured in the $45-450 \mathrm{keV}$ rest-frame band.

${ }^{\mathrm{b}}$ Completeness correction applied to scale the observed GRB counts to a common threshold of $E_{\text {iso }}>10^{51} \mathrm{erg}$.

${ }^{\mathrm{c}}$ Number of GRBs observed with $E_{\text {iso }}>E_{\text {iso,sens }}$.

d On-axis luminous $\left(E_{\text {iso,45-450 }}>10^{51} \mathrm{erg}\right)$ GRB rate density over this redshift interval, in units of $10^{-9} \mathrm{yr}^{-1} \mathrm{Mpc}^{-1}$.

the Gran Telescopio Canarias, the Inamori Magellan Areal Camera and Spectrograph (IMACS; Dressler et al. 2011) at Magellan, the Focal Reducer and Low Dispersion Spectrograph 2 (FORS2) at the VLT, the Wide Field Camera 3 (WFC3) aboard HST, and GROND (Greiner et al. 2008) at the MPG $2.2 \mathrm{~m}$ in La Silla.

In general, we make use of standard reduction techniques and pipelines where available to produce a stacked image, and then astrometrically align the stacked images against the Spitzer imaging. As the Spitzer post-basic calibrated data (PBCD) imaging is by default astrometrically aligned against the Two Micron All Sky Survey (2MASS) catalog (Skrutskie et al. 2006), this effectively establishes 2MASS as the astrometric reference system for the survey, and all positions reported in this work are therefore based on the 2MASS astrometric reference system.

Observational efforts are still ongoing (although nearly complete for targets above $\delta>-20^{\circ}$ ), so it is not yet possible to present a complete catalog of imaging acquired by the survey: this will be presented in forthcoming work following the completion of this effort. At the present time, we have collected over 690 individual photometric data points on host galaxies within the sample, of which more than 510 represent detections (the remainder being upper limits; nonconstraining upper limits due to poor weather are excluded from these numbers). These are supplemented by additional photometry from the literature. Every host galaxy except for one has at least one deep optical observation to supplement the IRAC data, and all except for 21 have at least one optical or infrared detection. Approximately half of the sample has numerous multicolor detections suitable for detailed characterization and modeling of the SED. We expect these statistics to improve modestly as our observational efforts wrap up during the coming year.

\subsection{Spectroscopic Observations}

A primary goal of the survey is to increase our spectroscopic completeness as high as is possible to remove any bias 
associated with redshift measurement-in particular, that associated with dark bursts, but also potentially for bursts that do have detectable afterglows that are fainter than average and more difficult if not necessarily impossible to obtain absorption spectra of in time (perhaps moderately extinguished bursts or those in low-density media).

Many of the events in our sample without an absorption redshift are prominent Swift dark bursts (or overlapped other uniformly constructed surveys, such as TOUGH or BAT-6), and as such a significant fraction of targets already had host galaxy redshifts in the literature, primarily from Jakobsson et al. (2012), Salvaterra et al. (2012), Krühler et al. (2012), Perley et al. (2013), or Krühler et al. (2015). All remaining sources showing bright ( $\lesssim 24 \mathrm{mag}$ in any band) host galaxy detections, and some fainter ones, were targeted for optical and/or NIR spectroscopy. For most of these observations we employ the X-shooter spectrograph at the VLT (Vernet et al. 2011), a medium-resolution cross-dispersed echelle spectrograph simultaneously covering the wavelength range between 0.3 and $2.5 \mu \mathrm{m}$. For observations associated with our program (094.A-0593, PI S. Schulze), four exposures of $900 \mathrm{~s}$ each were obtained in an ABBA nodding sequence and reduced in a standard manner using the $\mathrm{X}$-shooter pipeline provided by ESO (Goldoni et al. 2006) and using our own routines. For northern hemisphere targets, we used LRIS or MOSFIRE (McLean et al. 2012) on Keck I or NIRSPEC on Keck II and reduced the data using custom routines.

\subsection{Host Identification}

The number density of field galaxies on the sky is significant at the depths involved in our survey, so unambiguously identifying the host galaxy requires as accurate and reliable a position of the originating GRB as possible. Wherever possible, we acquired the original target-of-opportunity imaging files showing the optical or NIR transient, aligned these against the late-time host imaging, and recalculated the afterglow position, which provides astrometric accuracy of typically $0 . " 4$ or better (significantly smaller than the PSF of either the ground-based or Spitzer imaging). These images come from a number of sources, but common instruments are the imaging camera on the Palomar 60-inch telescope (Cenko et al. 2006b), the Nordic Optical Telescope, or publicly available guider camera imaging from VLT or acquisition exposures from Gemini. In some cases we downloaded imaging cutout figures posted in the GCN circulars or in published papers, and we recalculate the positions by aligning the cutouts to our host galaxy imaging in a similar fashion.

In cases where the original images are not available, we use astrometric coordinates published in the GCN circulars or in the literature, or supplied to us by others (in particular, by D. Malesani and Y. Urata). We apply an astrometric offset (measured directly from the USNO and 2MASS catalogs) where necessary to translate from a USNO-aligned system to 2MASS: we assume published optical coordinates to be in a USNO system if the astrometric system is not stated explicitly (except for NIR imaging, which we assume to be in the 2MASS system natively), although typically these offsets are quite small $(\sim 0$ !" $1-0$ ". 3$)$ and do not dominate the uncertainty. Radio or millimeter coordinates are not offset.

In some cases the only afterglow position available comes from Swift-typically from the XRT (as is often the case for dark GRBs), although in a few cases a Swift UVOT (UVOptical Telescope; Roming et al. 2005) position is available even though a ground-based position was not. Positions are taken from the automated XRT analyses of Butler \& Kocevski $(2007)^{27}$ and Evans et al. (2009) $)^{28}$, which provide positions with a typical accuracy of 1 !' 5 . UVOT positions are taken from the Swift GRB table. The coordinates are then shifted from their default USNO to the 2MASS frame, with the exception of positions noted as SDSS aligned in the Butler tables, which are not shifted.

Since in the vast majority of cases we do have a subarcsecond position available, the probability of mistaken identification of the host galaxy of any individual welllocalized GRB due to a chance foreground/background alignment is low-only approximately $1 \%$ of the sky is within 0 ". 4 of an unresolved $R<26 \mathrm{mag}$ galaxy (e.g., Hogg et al. 1997). Of course, in a large survey up to a few random alignments of this type would not be surprising but would not significantly affect the results of the survey. (Moreover, we can often identify and exclude them via a mismatch between host and afterglow redshifts; see Section 3.9). The XRT-only, 1"-2" positions are a source of somewhat greater concern, as the probability that a faint galaxy is present within a region of this size by chance is quite significant $(\sim 20 \%)$ and no prior redshift is available. This is partially alleviated by the fact that XRTonly events are typically dark, and that dark GRBs tend to originate from hosts that are much brighter than average (see, e.g., Perley et al. 2013 or Krühler et al. 2012) and have a low probability of chance association even considering the larger size of the XRT error circle. Only four sources have positional uncertainties greater than $1^{\prime \prime}$ and host galaxies fainter than $R=$ 25th magnitude $\left(P_{\text {chance }}>0.05\right)$ : GRB 050803, GRB 050922B, GRB 070621, and GRB 070808. Furthermore, the first two of these sources are associated with blue-dropout (likely, $z=4-5)$ galaxies, and the last is associated with an extremely red object-properties typical of optically faint (dusty or highz) GRB hosts but not common among galaxies selected randomly from the field.

\subsection{Host Galaxy Photometry}

Magnitudes (or upper limits) for host galaxies observed as part of our survey are measured using aperture photometry using a custom IDL wrapper around the aper photometric package included in the GSFC software library, or they are taken from the literature. Details of these procedures and the resulting photometry will be presented in future papers (Paper II, on the IRAC observations, is submitted concurrently with this paper) but are briefly summarized below. Photometry relevant to our photometric redshifts and upper limits are presented in Table 4.

IRAC images at the depths relevant to our survey are at or near the confusion limit, and most of our host galaxy targets show some degree of contamination from nearby foreground or background sources. To mitigate this contamination, we employ an iterative, partially automated PSF-fitting routine to model and subtract nearby objects identified from the Spitzer and ground-based imaging near the object and background apertures, leaving an uncontaminated image of the host. The host magnitudes are then measured via aperture photometry,

\footnotetext{
27 http://butler.lab.asu.edu/Swift/xrt_pos.html

28 http://www.swift.ac.uk/xrt_positions/index.php
} 


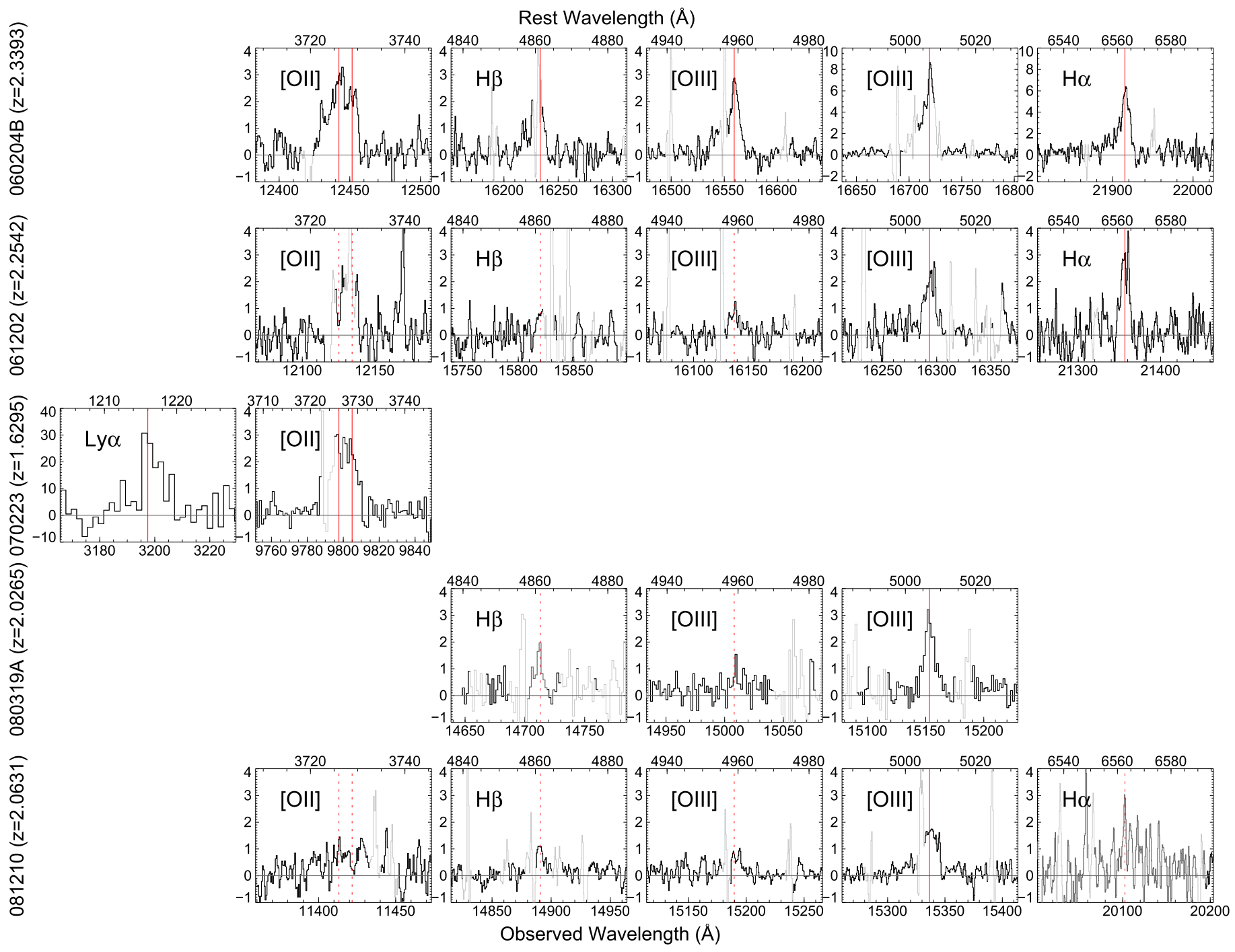

Figure 2. New secure spectroscopic host galaxy redshifts from our observations to date with VLT and Keck. Portions of the spectrum plotted in gray indicate wavelength regions affected by strong night-sky $\mathrm{OH}$ emission or by telluric absorption. (Many additional host spectroscopic redshifts are provided by our previously published work and from the literature in general; see Table 3.)

calibrated using the zero-point data in the IRAC handbook interpolated to the desired aperture using measurements of the instrumental PSF.

Photometry of optical/NIR images is provided in a similar manner, minus the need (in nearly all cases) to subtract contaminating sources since the host galaxy is well isolated. We calibrate relative to the latest release of SDSS (Ahn et al. 2014) wherever possible; otherwise, we provided our own secondary standards by observing the target on a photometric night alongside Landolt standards with a small telescope (P60 for northern targets or GROND for southern targets). Magnitudes in non-SDSS filters are interpolated from the available griz magnitudes using the photometric transformation equations of Lupton (2005). ${ }^{29}$ Calibration of HST photometry is performed using instrumental zero points.

\footnotetext{
${ }^{29}$ http://www.sdss.org/dr12/algorithms/sdssUBVRITransform/ \#Lupton2005
}

\section{NEW REDSHIFT CONSTRAINTS \\ 5.1. Host Galaxy Redshift Measurements}

Eight new (not previously published ${ }^{30}$ ) redshifts have so far been established by our program, either via the spectroscopic observations discussed in Section 4.3 or from photometric redshift fitting to the photometry we have gathered so far. A summary of new redshifts provided by these methods is given below. Zoom-ins of the spectral lines are presented in Figure 2, and our photometric SEDs (showing the EaZy model for the best-fit redshift) are shown in Figure 3.

GRB 050803 - The host galaxy is marginally $(3 \sigma-5 \sigma)$ detected in deep $R$-band imaging from the VLT, in $i$-band imaging from the GTC, and with the F160W ( $H$ band) filter on $H S T$, but no other wavelength (although a hint of a marginal detection is evident in the Spitzer imaging). The lack of $g$-band detection is indicative of a dropout at a redshift of $z \sim 4$,

\footnotetext{
${ }^{30}$ Some of these redshifts are also reported in Schulze et al. (2015) and Krühler et al. (2015), which were submitted close in time with this work by the same investigators.
} 


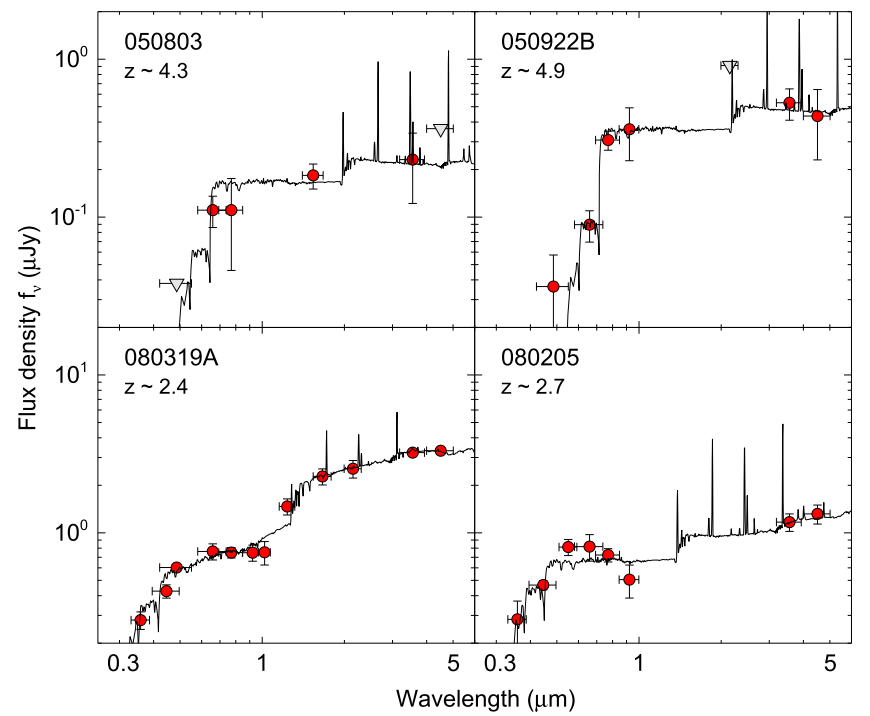

Figure 3. New photometric redshifts inferred by the survey. GRB 050803 and GRB 050922B show dropouts in the optical band indicative of a high redshift $z \sim 4-5$, although in the case of the former event this will need to be confirmed by deeper $g$-band imaging. The remaining events show a weaker flux decrement in the $U / B$ bands indicative of the onset of the Ly $\alpha$ forest and (for GRB 080319A) a Balmer jump in $J$ band at a consistent redshift. (The precise redshift of GRB 080319A was later fixed to $z=2.0265$ via emission-line spectroscopy.) The model SEDs shown are from EaZy at the best-fit photometric redshift.

although not an unambiguous one. Fitting the photometry using the EaZy photometric redshift software (Brammer et al. 2008) and disallowing passive low-redshift solutions indicates a redshift of $z \sim 4.3$ (with \pm 0.4 redshift uncertainty at $1 \sigma$ confidence, although $1.9<z<4.9$ is permitted at $95 \%$ confidence). This redshift is consistent with (but slightly higher than) the result of Schulze et al. (2015) using the same data (but different photo- $z$ software).

GRB 050922B-We observed this galaxy with LRIS ( $g$ and $R$ filters) and with OSIRIS ( $i$ and $z$ ), complementing the existing $R$ and $K_{s}$ imaging from the TOUGH survey. The host galaxy is well detected only in $i, z$, and in the Spitzer filters. A fit to the multifilter SED (again ruling out passive low- $z$ solutions) suggests that the redshift is high, $4.2<z<5.2$ with a best-fit value of $z=4.9$, also in agreement with the parallel analysis of Schulze et al. (2015).

GRB 060204B - X-shooter observations of the host galaxy of this GRB reveal many strong $(>8 \sigma)$ emission lines corresponding to $\mathrm{H} \alpha,[\mathrm{O} \mathrm{III}]_{\lambda 4959},[\mathrm{O} \mathrm{III}]_{\lambda 5007}$, and $[\mathrm{O} \mathrm{III}]_{\lambda 3727}$, as well as probable $\mathrm{H} \beta$, at a common redshift of $z=2.3393$. The velocity profile shows some structure with a prominent blue wing extending to about $300 \mathrm{~km} \mathrm{~s}^{-1}$.

GRB 061202-X-shooter observations of the host galaxy show strong emission lines consistent with $\mathrm{H} \alpha$ and $[\mathrm{O} \text { III }]_{\lambda 5007}$ at a common redshift of $z=2.2543$. Weaker emission is also seen at the locations of $[\mathrm{O} \text { III }]_{\lambda 4959}$ and $\mathrm{H} \beta$, and possibly $\left[\mathrm{O}_{\mathrm{II}}\right]_{\lambda 3727}$ (although the latter is in between two bright sky lines).

GRB 070223-Spectroscopy of the host galaxy with LRIS shows two strong emission lines at wavelengths of $\lambda=3198 \AA$ and $\lambda=9801 \AA$, corresponding to $\operatorname{Ly} \alpha$ and the $[\mathrm{O} \mathrm{II}]_{\lambda 3727}$ doublet at a common redshift of $z=1.6295$.

GRB 080319A-We imaged this field extensively with Keck/LRIS (UBgRiZ) and Keck/MOSFIRE (YJHK). The host is well detected in every band (and with IRAC). A fit to the SED indicates a photometric redshift of $z=2.43_{-0.36}^{+0.20}$. We also obtained $H$-band spectroscopy of this target using MOSFIRE on the Keck I telescope on 2015 June 07 UT. A total of 20 exposures of $120 \mathrm{~s}$ each were obtained. A single, unresolved emission line is visible in the subtracted and stacked 2D spectrum at a wavelength of $15153 \AA$. This could be either $[\mathrm{O} \mathrm{III}]_{\lambda 5007}$ at $z=2.0265$ or $\mathrm{H} \alpha$ at $z=1.309$, but the latter case is strongly ruled out by our photometric redshift. (An association with weaker lines such as $\mathrm{H} \beta$ or $[\mathrm{O} \mathrm{III}]_{\lambda 4959}$ is ruled out by the lack of additional line detections in the $H$-band spectroscopy.) We therefore infer a redshift of $z=2.0265$.

GRB 080205-A preliminary afterglow redshift of $z \sim 4.0$ was estimated by the UVOT team on the basis of an apparent dropout in the $B$ filter in the early-time UVOT photometry (Oates \& Markwardt 2008). However, we strongly detect the host in our ground-based $B$-band and $u$-band imaging, ruling out a redshift this high. A photometric fit to our $u B V R i z$ and Spitzer observations imposes a maximum redshift of $z<3.08$ with a best-fit redshift of $z \sim 2.7$ (with large uncertainties; $z=2.71_{-0.69}^{+0.25}$ ).

GRB 081210-X-shooter observations of this target show a strong emission line at $15336 \AA$ and some weaker features. We identify the line as $[\mathrm{O} \mathrm{III}]_{\lambda 5007}$ at $z=2.0631$ on the basis of probable $(4 \sigma)$ detections of $\mathrm{H} \beta$ and $[\mathrm{O} \mathrm{III}]_{\lambda 4959}$ at a consistent redshift and the fact that if this were another strong line (e.g., $\mathrm{H} \alpha$ ), other lines should be detected in clean regions of the spectrum but are not observed. At $z=2.0631$ the wavelength corresponding to $\mathrm{H} \alpha$ is in a region of moderately strong telluric absorption.

\subsection{Redshift Upper Limits}

Nine sources have thus far eluded redshift measurement. However, even in most of these cases we can place upper limits owing to the detection of optical afterglow, the detection of significant soft X-ray absorption excess above the foreground Galactic value (Grupe et al. 2007), or the detection of an optical host galaxy.

GRB 050128 - We retrieved deep archival FORS1 imaging of this source from the VLT archive; the data (associated with program 075.A-0718(A)) were taken on 2005 May 11 UT and for a total $3080 \mathrm{~s}$ of exposure time in the $R$-band filter. Within the XRT error circle we securely detect a host galaxy candidate in the combined stack. The detection in this band indicates $z \lesssim 5.5$.

GRB 050726 - The host galaxy is not detected in a very deep VLT $R$-band observation taken as part of the TOUGH project, so the host cannot be used to constrain the redshift. However, a detection of an early afterglow was reported in the initial UVOT $V$-band exposure, suggesting $z<3.5$. Nondetections in subsequent UVOT $B$ - and $U$-band observations suggest that the GRB may be a dropout and therefore close to this maximum redshift, but it is also quite possible that the GRB faded rapidly (Poole et al. 2005) — so only an upper limit can be placed.

GRB 070621 - The (probable) host of this object, first identified in TOUGH, is very faint and detected only in $R$ band; while observations have been acquired in other filters with Keck and Gemini, they do not reach the depths needed to detect the host. The afterglow was not detected at any optical 


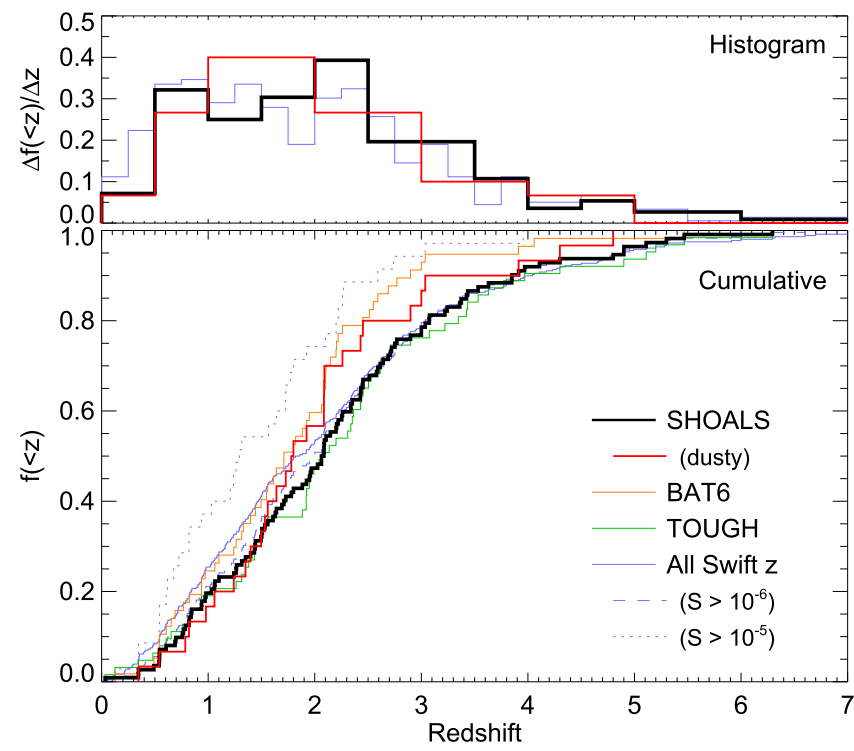

Figure 4. Redshift distribution of SHOALS GRBs (solid black line) compared to other GRB samples. Targets are mostly between $0.6<z<4.0$, with a few events at lower and higher redshifts; the overall distribution closely resembles that of known-redshift Swift GRBs, as well as other complete GRB samples (e.g., TOUGH). Our modest fluence cut $\left(S_{15-150 \mathrm{keV}}>10^{-6} \mathrm{erg} \mathrm{cm}^{-2}\right)$ does not greatly affect the redshift distribution compared to that of Swift GRBs.

band despite deep early-time imaging. The absorbing column inferred from the XRT spectrum is large, although not definitively so, and it places only a weak redshift limit of $z<5.5$ (similar to what is implied by the putative $R$-band host detection).

GRB 070808 - The afterglow of this event was detected only by XRT, and the identity of the host galaxy itself is subject to some ambiguity given the coarse position; the nearest source to the center of either the DSS-refined or UVOT-refined XRT error circles was previously suggested by Hjorth et al. (2012) as the most likely host candidate. This putative host galaxy is very red and well detected in IRAC and in VLT $K_{s^{-}}$ band imaging but only marginally or not at all in the deep optical imaging acquired so far. Our optical photometry is not sufficient to establish a reliable photometric redshift on its own, but the red 3.6-4.5 $\mu \mathrm{m}$ color effectively rules out a low-redshift solution (requiring $z>0.5$ ). This GRB also has one of the largest XRT $N_{H}$ equivalent column excesses measured in the entire sample, which in this case actually places a more constraining limit on the redshift than any properties of the host galaxy. Using the lower-limit $N_{H}$ value measured from latetime XRT data by Butler \& Kocevski (2007) of 4.06 $\times 10^{21} \mathrm{~cm}^{-2}$ places a maximum redshift of $z<2.2$ according to the empirical formula of Grupe et al. (2007). ${ }^{31}$ Treating the upper and lower limits together provides a crude redshift estimate of $z=1.35 \pm 0.85$.

GRB 081128 - A bright galaxy is located at the edge of the XRT error circle, although its centroid is significantly offset

\footnotetext{
31 To critically assess the Grupe et al. (2007) relation, we repeated this procedure on the entire catalog of Swift events with spectroscopic redshifts, including dark bursts. With the exception of a handful of events with unrealistically large minimum $N_{H}$ values $\left(\sim 10^{25} \mathrm{~cm}^{-2}\right.$, probably due to intrinsic curvature or difficulty with the automated model fitting; at face value these would actually imply negative redshifts from the Grupe relation), we found only a single event with a redshift in excess of the predicted value: GRB 080207 (which exeeded it by only $\Delta z=0.01$ ).
}

$\left(\sim 1^{\prime \prime}\right)$ from the optical afterglow position. A combination of multifilter imaging and spectroscopy from both LRIS and $\mathrm{X}$-shooter shows it to be an early-type galaxy at $z=0.27$ with very little star formation. In addition, a much fainter source is evident directly underlying the afterglow in LRIS $B-, g$-, and $V$ band imaging (and marginally in $i$ - and $z$-band imaging with the same instrument and with IRAC). While the nature of this system is not completely clear, the significant separation and color differential suggest that the bright galaxy is a foreground system unrelated to the GRB and the faint, blue object represents the true host galaxy. We fit the photometry of the fainter source using EaZy; while a consistent redshift of $z=0.32$ is marginally favored, higher-redshift solutions (in particular, $z \sim 3$ ) are also credible. We can place only an upper limit of $z<3.4$ on the redshift.

GRB 100305A-No host galaxy is detected at the XRT position in our imaging (we note that the proposed optical counterpart of Cucchiara [2010] is well outside the final XRT error circle and the source is still present in our own Keck imaging, so it is not likely to be associated with the GRB). Two objects are just outside the edge of the error circle: one, to the southeast, is seen only in Spitzer and appears extended; the other, to the southwest, is also detected in our optical imaging. The uncertainty about the host identification and the lack of optical afterglow precludes a definitive upper limit based on these data. The excess X-ray column is also low, suggesting that this may indeed be a high-redshift event that was "missed" on the basis of its faint afterglow. This is the only event in the sample without an upper limit on its redshift.

GRB 100802A - The only reported detection of the optical afterglow is by P60 (Cenko 2010). Only an $r$-band detection was reported originally. We stacked all of the imaging of the GRB acquired by the telescope that night and recovered detections in all four griz bands and aligned these images against our late-time Keck imaging. A source is clearly detected in the Keck images at this location in $B$ band and (marginally) in $R$ and $i$ band, and with IRAC. The data are not sufficient to estimate a definite photometric redshift but place an upper limit of $z<3.1$.

GRB 110709B-The host galaxy (first reported by Zauderer et al. 2013) was observed with X-shooter (under VLT Program 090.A-0088), integrating for $8 \times 900 \mathrm{~s}$ using the $K$-blocking filter. The reduced $2 \mathrm{D}$ frame does not show any strong lines over the spectral range, although a weak $(5 \sigma)$ line candidate is seen at an observed wavelength of $15479 \AA$. The most likely identification of this feature is $[\mathrm{O} \mathrm{III}]_{\lambda 5007}$ at a redshift of $z=2.091$, as other strong-line matches are ruled out $\left([\mathrm{O} \mathrm{II}]_{\lambda 3727}\right.$ would be resolved, while $\mathrm{H} \alpha$ would imply emission lines of $[\mathrm{O} \text { III }]_{\lambda 5007}$ and $[\mathrm{O} \mathrm{II}]_{\lambda 3727}$ within regions of the spectrum where we have good sensitivity and do not observe). We consider this assignment tentative pending future follow-up without the $K$-blocking filter. Photometrically, the redshift can be limited only to $z \lesssim 5.5$ (based on the HST F606W host detection).

GRB 120308A-The host is securely detected in our LRIS $g$-band and $R$-band imaging from 2014 June 23, as well as in $I$ band LRIS imaging from 2014 May 27 and with IRAC. We attempted a photometic redshift fit to these data; this is unable to produce a lower limit on the redshift but does establish an upper limit of $z<3.7$. 


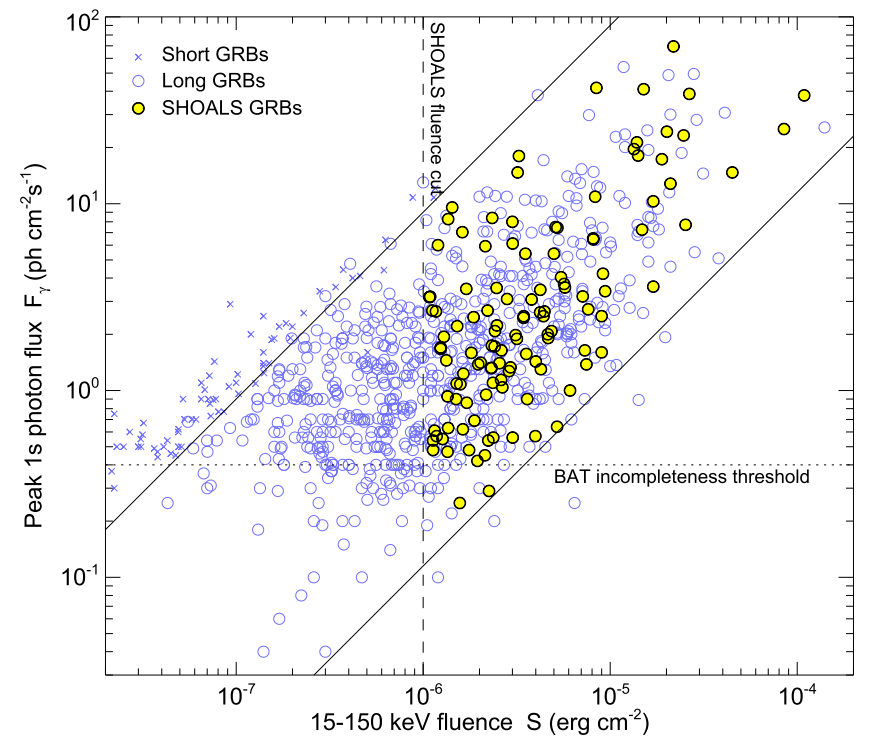

Figure 5. Peak photon flux vs. total fluence for Swift GRBs. Events in our sample are shown in yellow. Although the BAT triggers (approximately) according to photon count rate and our sample is cut in fluence, owing to the correlation between the two parameters, our sample nevertheless is nearly complete in fluence: only the small triangular region to the right of the fluence cut and below the incompleteness threshold is expected to contain real bursts that would have passed our fluence cut if they had successfully triggered the BAT. Diagonal lines are of constant $F_{\gamma} / S$ and show the region containing nearly all long GRBs.

\section{RESULTS}

\subsection{Redshift Distribution}

The cumulative redshift distribution of our sample, as well as a binned fractional histogram, is plotted in Figure $4 .^{32}$ Remarkably, the redshift distribution of the sample is very similar to that of the overall Swift distribution. This suggests that, in spite of the many biases potentially affecting redshift measurement for a typical Swift GRB, the net impact on the redshift distribution is small.

We investigated this more closely by calculating the redshift distributions of the dust-obscured GRBs and other GRBs separately (the most prominent instrinsic factor affecting redshift incompleteness is dust obscuration, which makes afterglow-based redshift determination for $\sim 20 \%$ of GRBs impossible in practice). The redshift distribution for obscured bursts is shown as a red line in Figure 4. Its distribution closely mirrors the general SHOALS population: a mild excess of GRBs is seen at $1.5<z<2.5$, but a K-S test suggests that this is not particularly significant ( $p=0.15$ comparing the obscured GRBs in the sample vs. the remaining GRBs with redshifts). This suggests that the fraction of cosmic star formation that is obscured does not vary by a large amount with redshift, at least over the range in which we have reasonable number statistics $(1<z<4)$.

\footnotetext{
${ }^{32}$ Here and in subsequent analysis we place photometric redshifts (there are only six) at their best-fit values and also use our best-guess redshift values for GRB 070808 and GRB 110709B. The remaining unknown- $z$ bursts are omitted. Removing photometric/insecure host redshifts would slightly decrease (by $\sim 15 \%$ ) the fraction of $z \sim 3.5-5.5$ events in the sample and have negligible impact on the rest of the distribution; this would not influence our results/conclusions. If all unknown- $z$ events were very close to their maximum redshift, their inclusion would increase the fraction of $z \sim 3.5-5.5$ events slightly (by $\sim 20 \%$ ) and likewise have neglible impact on the rest of the distribution; again, this would not affect our results or conclusions.
}

The redshift distribution is also very similar to that of the TOUGH sample (a similar unbiased sample-but with no fluence cut), indicating that, even allowing for the significant overlap between the samples, the fluence cut we employed has only a relatively minor impact. In contrast, the BAT-6 sample (which is cut fairly stringently on peak flux) shows a notable skew in its redshift distribution toward lower redshifts. While our study is not contingent on the redshift distribution of our sample matching that of Swift GRBs overall (indeed, we would expect some differences based on the arguments in Section 3.6), this indicates that our final sample is nevertheless reasonably representative of the broader Swift population in redshift distribution.

We observe a very small fraction of the sample at high redshifts. Only a single event is confirmed to be at $z>5.5$ (GRB 050904 at $z=6.295$ ), and among the nine GRBs in the sample with no measured redshift, all but one is limited to $z<5.5$ - so at most two events out of the 119 in our sample can be at $z>5.5$. This infrequency is qualitatively consistent with other complete studies (e.g., Fynbo et al. 2009b; Perley et al. 2009c; Greiner et al. 2011; Jakobsson et al. 2012; Salvaterra et al. 2012) but even more constraining. Repeating the Monte Carlo analysis technique of Perley et al. (2009c) on our sample, we estimate that intrinsically between $0.3 \%$ and $5 \%$ of $S>10^{-6} \mathrm{erg} \mathrm{cm}^{-2}$ Swift GRBs can originate at $z>5.5$ ( $95 \%$ confidence).

While it is possible that we are preferentially missing GRBs at $z \gtrsim 6$ in SHOALS relative to the all-Swift sample owing to the fluence cut, as discussed earlier in this section, the impact of a fluence cut at $10^{-6}$ on the redshift distribution appears to be small in practice. Our results therefore suggest that the high- $z$ rate for Swift bursts may be even lower than previously suspected (by, e.g., Perley et al. 2009c): a few events per year. This underscores the challenges faced by recent efforts to observe high-redshift GRBs and helps to explain the recent paucity of confirmed high- $z$ events. Likewise, it contributes to our finding that the intrinsic high- $z$ GRB rate density is somewhat more modest than earlier estimates (Section 6.3).

\subsection{Sample Completeness with Respect to Prompt Emission Properties}

While we elected to cut on fluence ${ }^{33}$ for the reasons detailed in Section 3.6, Swift's primary trigger mechanism is more strongly tied to a burst's peak flux, and a difficult-to-quantify incompleteness affects the satellite's ability to detect and trigger on bursts with peak flux close to its thresholdcomplicating, in principle, any attempts to measure the intrinsic GRB rate. While a cut on peak flux at a value above the incompleteness level would largely eliminate this concern, the impact of our fluence cut is less straightforward: it is possible that Swift itself may have missed some GRBs whose fluence was above our cut level but whose peak flux was too low to trigger the instrument (owing to a particularly long and smooth light curve).

Even so, we have reason to expect that the sample established by our fluence cut is nearly complete. In Figure 5

\footnotetext{
33 Here and throughout the paper, we emphasize that the flux and fluence we refer to as measured refer only to the BAT $15-150 \mathrm{keV}$ band, not broadband or bolometric values. Because GRBs occupy a wide range of intrinsic $E_{\text {peak }}$, there are many GRBs with bolometric fluences well above our cut that BAT is insensitive to, but because we consider BAT-band properties alone, this does not impact our analysis or conclusions.
} 


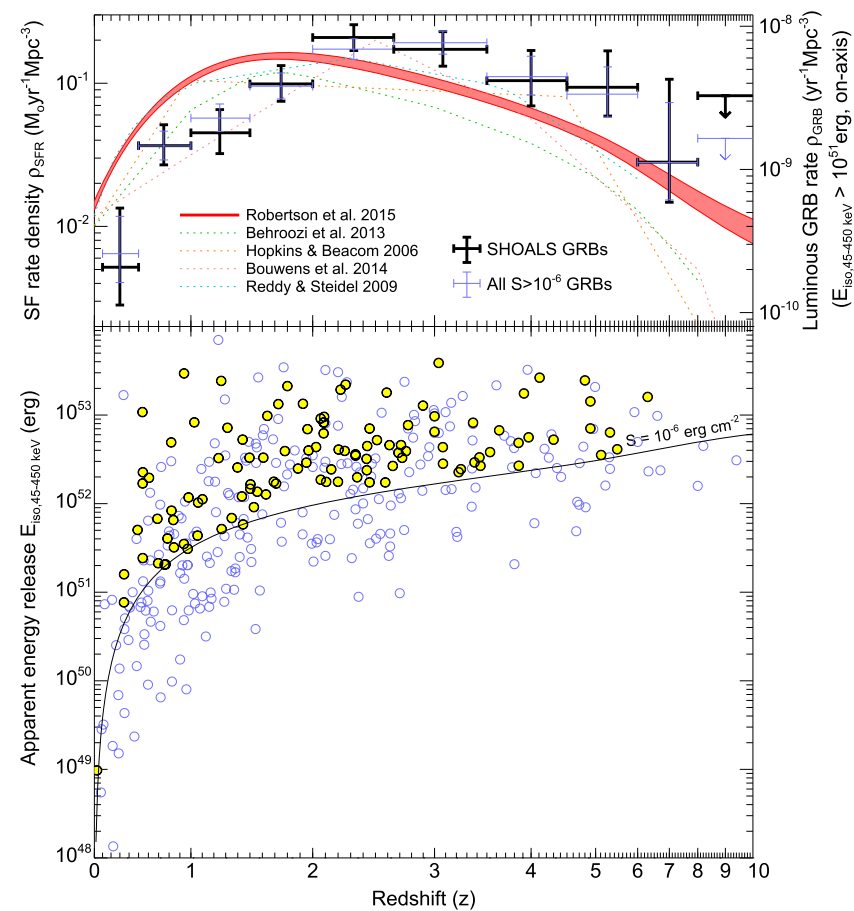

Figure 6. Comoving GRB rate density vs. redshift compared to the comoving field-survey SFR density (top panel), as inferred from our redshift and $E_{\text {iso }}$ distribution (lower panel). Curves plot field-galaxy star formation histories from various sources (Madau \& Dickinson 2014 via Hopkins \& Beacom 2006; Reddy \& Steidel 2009; Robertson \& Ellis 2012; Behroozi et al. 2013; Bouwens et al. 2014; Robertson et al. 2015). The scaling of the GRB rate (thick data points from the SHOALS sample, thin measurements from all $S>10^{-6} \mathrm{erg} \mathrm{cm}^{-2}$ Swift GRBs; error bars denote $10 \%-90 \%$ binomial confidence interval) is arbitrary, normalized against the SFR at $z \sim 2$. The behavior of the GRB rate history is qualitatively similar to the SFR history, but shows a modest (factor of $\sim 5$ ) excess at high redshifts (or equivalently, a depression at low redshift) compared to the most recent SFR density measurements.

we plot the peak photon flux and total measured energy fluence for all Swift GRBs (open circles) and for our SHOALS sample (filled circles). ${ }^{34}$ Unsurprisingly, these two parameters strongly correlate in a linear fashion but show scatter at the level of $\sim 1$ dex; the region of flux-fluence space inhabited by most Swift long-duration GRBs (98\%) is demarcated by the solid diagonals. The effective BAT triggering threshold is readily apparent in the data at approximately 0.4 photons $\mathrm{cm}^{-2} \mathrm{~s}^{-1}$. For bursts with very high fluences, the BAT trigger sensitivity is clearly not an issue (e.g., no bursts with a fluence of $S>10^{-5} \mathrm{erg} \mathrm{cm}^{-2}$ have a peak flux anywhere near the BAT incompleteness threshold: to a good approximation, bursts this bright in the field of view should always trigger the telescope regardless of light-curve shape, off-axis angle, etc.). For our chosen cut level of $S>10^{-6} \mathrm{erg} \mathrm{cm}^{-2}$, instrumental incompleteness is not completely negligible, and a few events that in principle may have been bright enough to meet our selection criteria could have been missed by the BAT. Based on the flux/ fluence ratio distribution for brighter bursts where we are confident that the BAT sample is complete, we expect that the number of such events is relatively small ( $\sim 10$ or less) and not

\footnotetext{
34 Figure 5 and subsequent figures/tables show the updated (Sakamoto et al. 2011) values of flux and fluence for bursts where revised measurements of both values are available. Our results do not differ if the original GCN measurements are used.
}

likely to have a significant impact on the conclusions presented in this paper.

\subsection{The Redshift-dependent GRB Rate Density}

The observed GRB redshift distribution can be used to infer the intrinsic comoving GRB rate, provided that the sensitivity of the instrument and selection of the known-redshift sample are well understood and the GRB luminosity function can also be inferred. As we argue above that the sample is intrinsically fluence limited to a good approximation, these procedures can be applied to our sample also (using energetics-dependent quantities in place of the more standard luminosity-dependent quantities).

We first calculate the isotropic-equivalent energy releases for all GRBs in our sample, $E_{\mathrm{iso}, 45-450 \mathrm{keV}}=S_{15-150 \mathrm{keV}}$ $4 \pi d_{L}^{2}(1+z)^{-1} k(z)$ (e.g., Bloom et al. 2001). Unlike most previous authors, we do not make any attempt to estimate a bolometric or wide-bandwidth value, which is fraught with uncertainty considering that Swift's bandpass is narrow and does not usually contain the peak energy of the burst; the BATto-bolometric correction can easily be orders of magnitude, and it is practically unconstrained by the Swift data. Instead, we calculate the energy release in the $45-450 \mathrm{keV}$ rest-frame band only, corresponding to the window observed by the BAT (15-150 keV observer-frame) for an event at $z \sim 2$, close to the approximate sample redshift median and also where previously reported divergences in the GRB-to-SFR ratio begin to become apparent. This makes the $k$-correction much smaller and more reliable: specifically, its value is $k(z)=$ $[(1+z) /(1+2)]^{\Gamma-2}$, where $\Gamma$ is the measured photon index over the BAT band (for bursts in which $\Gamma$ is not well constrained by the data we take the population median value of $\Gamma=1.5$ ). This $k$-correction is always small (exactly unity at $z=2$ and within a factor of 2 between $1<z<4$ for any common value of $\Gamma$ ), and the uncertainty is even smaller (typically a few percent), so these measurements should be highly reliable.

We assume an intrinsic isotropic-equivalent energetics rate function $\phi\left(E_{\text {iso }}\right)$ following a single power law and measure its power-law index by fitting the observed fluence distribution of GRBs at moderate redshift $(0.5<z<3.5)$ in the entire Swift sample above our fluence cut; we infer $\phi_{E \text { iso }} \propto E_{\text {iso }}^{-1.7 \pm 0.2}$. We then count the observed number of GRBs (in both the SHOALS sample and for all $S>10^{-6}$ Swift GRBs) with $E_{\text {iso }}>10^{51} \mathrm{erg}$ per redshift bin and use the integral of the energetics function to correct the number in each bin for incompleteness in $E_{\text {iso }}$, scaling all bins to a common energy cutoff of $E_{\text {iso }}>10^{51} \mathrm{erg}$. This number is then scaled in the usual way by $d V / d z /(1+z)$ to convert the density in redshift to a comoving rate density.

The resulting redshift-dependent rate (top panel of Figure 6 and Table 3) shows a broad peak at $z \sim 1.5-3.5$ and a modest decline toward lower and higher redshifts. This behavior is consistent with previous studies of this type (e.g., Kistler et al. 2008, 2009; Butler et al. 2010a; Wanderman \& Piran 2010; Jakobsson et al. 2012; Robertson \& Ellis 2012; Salvaterra et al. 2012)_but, unlike these previous studies (with the exceptions for the significantly smaller samples of Jakobsson et al. 2012 and Salvaterra et al. 2012), our results are not limited by systematics associated with the highly incomplete redshift distribution. 
It is somewhat surprising that the complete, unbiased redshift distribution produced from our work is so similar to the distribution inferred from previous studies based (largely) on samples drawn from afterglow redshifts in the literature, which favor low-extinction sightlines. As we mentioned earlier (Section 6.3), this suggests a relatively uniform fraction of obscured star formation across most of cosmic history (our sample provides good number statistics between approximately $0.5<z<4)$. It also indicates that high- $z$ GRBs, despite being uncommon (intrinsically and observationally) and difficult to follow up, are being identified with comparable efficiency as their low-redshift counterparts.

\section{A REDSHIFT-DEPENDENT GRB EFFICIENCY?}

Since GRBs originate from massive stars, the comparison of the GRB rate history and the SFR history as derived by traditional galaxy survey methods (e.g., Madau et al. 1998; Hopkins \& Beacom 2006; Reddy \& Steidel 2009; Behroozi et al. 2013; Bouwens et al. 2014; see Madau \& Dickinson 2014 for a review) imposes a constraint on the degree to which the GRB rate is affected by other factors, as well as an independent check on the SFR history itself. Many previous studies have quantitatively compared these two relations in detail; most of these (Daigne et al. 2006; Guetta \& Piran 2007; Le \& Dermer 2007; Salvaterra \& Chincarini 2007; Kistler et al. 2008; Yüksel et al. 2008; Virgili et al. 2011; Robertson \& Ellis 2012; Lien et al. 2014; see. Wanderman \& Piran 2010; Elliott et al. 2012; $\mathrm{Yu}$ et al. 2015) have concluded that the GRB rate at high redshift is significantly higher than what would be inferred from the galaxy-survey-inferred star formation history. This suggested either that the field surveys were insufficiently accounting for the number density of low-luminosity galaxies they do not detect, or that the cosmic GRB production efficiency $\epsilon(z)=\rho_{\mathrm{GRB}}(z) / \rho_{\mathrm{SFR}}(z)$ is not constant (owing to, e.g., metallicity enrichment suppressing the GRB rate at lower redshift; see, e.g., Jakobsson et al. 2012; Trenti et al. 2013).

We carried out this exercise with our own observations as well, comparing our (selection-unbiased) rate distribution with the most recent estimate of the SFR density out to very high redshifts using the recent galaxy luminosity functions of Madau \& Dickinson (2014), integrated down to $L=10^{-3} L_{*}$ galaxies by Robertson et al. (2015). We confirm the high-redshift excess (or, equivalently, a low-redshift deficiency) in the GRB rate relative to the UV-inferred SFR: normalizing ${ }^{35}$ the two curves at $z=2$, the $z \sim 5$ GRB rate is in excess of the SFR by a factor of 2-3, and the $z<0.5$ GRB rate is below it by a factor of 2-5.

This provides further support to the notion that the cosmic GRB efficiency may vary over time. We emphasize, however, that while the deviation is significant, it is also relatively modest in magnitude: a factor of $\sim 5-10$ across the entire span of cosmic history from $z=5$ to $z \sim 0$. In fact, perhaps the more salient conclusion to be drawn from Figure 6 is that strong evolution in the GRB-to-SFR ratio is not observed. This argues that the GRB rate's dependency on metallicity must have only low to moderate impact on the cosmological rate, in disagreement with models implying strong variations (e.g., the singlestar models of Langer \& Norman [2006], which require approximately $Z<0.1 Z_{\odot}$, would imply a variation by more

\footnotetext{
35 Note that the relative normalization of the two curves is effectively arbitrary since we do not know the fraction of stars that explode as GRBs or the beaming correction to better than an order of magnitude.
}

than a factor of 40 over this period). Earlier analyses using nonuniform afterglow-based samples (Robertson \& Ellis 2012; Hao \& Yuan 2013; Trenti et al. 2013) have reached similar conclusions but were limited by systematics associated with incomplete redshift measurement; our work confirms that these conclusions hold within an unbiased sample.

Our result is also in agreement with independent investigations of the variations of the GRB rate based on the properties of the hosts themselves. Recent emission-line studies (e.g., Graham \& Fruchter 2013; Krühler et al. 2015) show that GRBs form readily at moderate metallicities $\left(\sim 0.5 Z_{\odot}\right.$ or more $)$ that are characteristic of typical star-forming galaxies throughout most of the universe's history. And the host stellar mass distribution we infer from IRAC observations of the host galaxies in our sample ( Paper II) similarly suggests that a metallicity threshold for GRB production is likely present but is relatively high, $\sim Z_{\odot}$.

\section{SUMMARY}

We have defined a new legacy sample of GRBs and host galaxies spanning nearly all of cosmic history: 119 Swift GRBs at $0.03<z<6.29$ drawn from the Swift GRB catalog using a series of observability cuts, plus a requirement that the BAT fluence exceed $S>10^{-6} \mathrm{erg} \mathrm{cm}^{-2}$. Combining publicly available afterglow and host galaxy redshift measurements with our own host galaxy campaign, we present redshifts for $110(92 \%)$ of these bursts, or $112(94 \%)$ if we include the tentative redshift of GRB 110709B and the lower+upper limits on GRB 070808. This is by far the largest, and the most redshift-complete, sample of its type, and it provides the most up-to-date and unbiased view of cosmic history as seen by GRBs. Only one event lacks a redshift upper limit, and only one event with measured redshift is at $z>5.5$.

Mapping our redshift distribution to a comoving rate density to measure the evolution of the GRB rate with cosmic time, we measure a rise in the GRB rate from $z>6$ to $z \sim 2$, followed by a drop of an order of magnitude from $z \sim 2$ to the present time-the same pattern seen by traditional metrics of the cosmic SFR density. Quantitatively comparing the GRB rate history to the cosmic star formation history, we find a modest excess in the GRB rate (versus SFR density) at high redshift compared to lower redshifts. Consistent with previous work, this suggests that the cosmic GRB efficiency was higher in the first few billion years of cosmic history relative to today and provides support to the idea of a metal-dependent progenitor, but the modest degree of this variation rules out models requiring an exclusively very metal-poor (e.g., $<0.1 Z_{\odot}$ ) environment. The small number of high-redshift GRBs in the sample places strong limits on the fraction of high- $z$ bursts detected by Swift and on the intrinsic GRB rate at high redshifts.

Addressing the GRB rate-evolution question in detail-and actually applying our GRB population to address broader questions in astronomy-requires more than just redshifts. In particular, examination of the afterglows and (especially) host galaxies of these events is needed to study the galaxy population giving rise to the GRBs in our sample at each redshift, providing an independent test of factors controlling the GRB rate and a means to explore directly the importance and nature of the dusty, low-luminosity, and high-redshift populations uniquely probed by GRBs. We are collecting and analyzing these observations under the programs introduced here, and the direct study of the 
hosts within our sample will serve as the subject of all remaining papers. As the first large, thorough, highly complete, and multiband survey of an unbiased GRB host sample, SHOALS will enable unprecedented insight into the GRB rate and progenitor, as well as a unique perspective into galaxy evolution and cosmic history.

This work is based in part on observations made with the Spitzer Space Telescope, which is operated by the Jet Propulsion Laboratory, California Institute of Technology, under a contract with NASA. It is also based in part on observations with the NASA/ESA Hubble Space Telescope, obtained from the Space Telescope Science Institute. STScI is operated by the Association of Universities for Research in Astronomy, Inc., under NASA contract NAS 5-26555. These observations are associated with program GO-90062. Support for this work was provided by NASA through an award issued by JPL/Caltech and through Hubble Fellowship grant HST-HF-51296.01-A awarded by the Space Telescope Science Institute, which is operated by the Association of Universities for Research in Astronomy, Inc., for NASA, under contract NAS 5-26555. D.A.P. further acknowledges support from a Marie Sklodowska-Curie Individual Fellowship within the Horizon 2020 European Union (EU) Framework Programme for Research and Innovation (H2020MSCA-IF-2014-660113). The Dark Cosmology Centre is funded by the DNRF. The research leading to these results has received funding from the European Research Council under the European Union's Seventh Framework Program (FP7/ 2007-2013)/ERC Grant agreement no. EGGS-278202. S.S. acknowledges support from CONICYT-Chile FONDECYT 3140534, Basal-CATA PFB-06/2007, and Project IC120009 "Millennium Institute of Astrophysics (MAS) of Iniciativa Científica Milenio del Ministerio de Economía, Fomento y Turismo. S.K. acknowledges support from FONDECYT 3130488. A.C. is supported by the NASA Postdoctoral Program at the Goddard Space Flight Center, administered by Oak Ridge Associated Universities through a contract with NASA. A.d.U.P. and C.C.T. are supported by Ramón y Cajal fellowships.

Some of the data presented here were obtained at the W. M. Keck Observatory, which is operated as a scientific partnership among the California Institute of Technology, the University of California, and the National Aeronautics and Space Administration. The Observatory was made possible by the generous financial support of the W. M. Keck Foundation. The authors wish to recognize and acknowledge the very significant cultural role and reverence that the summit of Mauna Kea has always had within the indigenous Hawaiian community. Based on observations made with ESO Telescopes at the La Silla Paranal Observatory and on observations made with the Gran Telescopio Canarias (GTC). Part of the funding for GROND (both hardware and personnel) was generously granted from the Leibniz-Prize to Prof. G. Hasinger (DFG grant HA 1850/28-1).

It is a pleasure to thank the Swift team for creating such a superbly prolific and successful instrument, without which this study would have been impossible. We also wish to extend thanks to the entire ground-based GRB follow-up community for providing many of the afterglow identifications and redshifts critical to our study. We particularly acknowledge D. Malesani, Y. Urata, and K. Huang for providing improved afterglow positions and images, and we thank the anonymous referee and D. A. Kann for useful comments. We also thank our other collaborators for assistance with acquiring ground-based observations, including R. Sanchez Ramirez, F. E. Bauer, and P. Schady. We thank B. Robertson and M. Trenti for useful discussions and also thank B. Robertson for providing the most up-to-date SFR density curves.

Facilities: Spitzer:IRAC, Keck-I:LRIS, Keck-I:MOSFIRE, HST:WFC3, VLT:X-shooter, GTC:OSIRIS, Max Planck:2.2 m (GROND), PO:1.5 m.

\section{REFERENCES}

Ahn, C. P., Alexandroff, R., Allende Prieto, C., et al. 2014, ApJS, 211, 17 Akerlof, C. W., \& Swan, H. F. 2007, ApJ, 671, 1868

Band, D. L. 2006, ApJ, 644, 378

Barthelmy, S. D., Barbier, L. M., Cummings, J. R., et al. 2005, SSRv, 120, 143 Behroozi, P. S., Wechsler, R. H., \& Conroy, C. 2013, ApJ, 770, 57 Berger, E. 2014, ARA\&A, 52, 43

Berger, E., Cowie, L. L., Kulkarni, S. R., et al. 2003, ApJ, 588, 99 Berger, E., Foley, R., Simcoe, R., \& Irwin, J. 2008, GCN Circ., 8434 Berger, E., \& Fox, D. B. 2009, GCN Circ., 9156

Berger, E., \& Rauch, M. 2008, GCN Circ., 8542

Berger, E., Kulkarni, S. R., Bloom, J. S., et al. 2002, ApJ, 581, 981 Berger, E., Kulkarni, S. R., Fox, D. B., et al. 2005, ApJ, 634, 501 Blain, A. W., \& Natarajan, P. 2000, MNRAS, 312, L35

Bloom, J. S. 2007, GCN Circ., 6989

Bloom, J. S., Frail, D. A., \& Sari, R. 2001, AJ, 121, 2879

Bloom, J. S., Perley, D. A., \& Starr, D. L. 2007, GCN Circ., 6953

Bloom, J. S., Perley, D. A., Li, W., et al. 2009, ApJ, 691, 723

Boissier, S., Salvaterra, R., Le Floc'h, E., et al. 2013, A\&A, 557, A34

Bouwens, R. J., Bradley, L., Zitrin, A., et al. 2014, ApJ, 795, 126

Brammer, G. B., van Dokkum, P. G., \& Coppi, P. 2008, ApJ, 686, 1503

Bromberg, O., Nakar, E., \& Piran, T. 2011, ApJL, 739, L55

Bromm, V., \& Loeb, A. 2006, ApJ, 642, 382

Burrows, D. N., Hill, J. E., Nousek, J. A., et al. 2005, SSRv, 120, 165

Burrows, D. N., Kennea, J. A., Abbey, A. F., et al. 2007, Proc. SPIE, 6686, 7

Butler, N. R. 2007, AJ, 133, 1027

Butler, N. R., Bloom, J. S., \& Poznanski, D. 2010a, ApJ, 711, 495

Butler, N. R., \& Kocevski, D. 2007, ApJ, 663, 407

Butler, N. R., Perley, D. A., Cenko, S. B., et al. 2010b, GCN Circ., 10915

Cameron, E. 2011, PASA, 28, 128

Campisi, M. A., Maio, U., Salvaterra, R., \& Ciardi, B. 2011a, MNRAS, 416, 2760

Campisi, M. A., Tapparello, C., Salvaterra, R., Mannucci, F., \& Colpi, M. 2011b, MNRAS, 417, 1013

Castro-Tirado, A. J., de Ugarte Postigo, A., Jelinek, M., et al. 2007, GCN Circ., 6971

Castro-Tirado, A. J., Bremer, M., Winters, J.-M., et al. 2009, GCN Circ., 9100

Cenko, S. B. 2005, GCN Circ., 3807

Cenko, S. B. 2008, GCN Circ., 7429

Cenko, S. B. 2009, GCN Circ., 9769

Cenko, S. B. 2010, GCN Circ., 11040

Cenko, S. B., Berger, E., Djorgovski, S. G., Mahabal, A. A., \& Fox, D. B. 2006a, GCN Circ., 5155

Cenko, S. B., \& Fox, D. B. 2006, GCN Circ., 5978

Cenko, S. B., Hora, J. L., \& Bloom, J. S. 2011, GCN Circ., 11638

Cenko, S. B., Perley, D. A., Junkkarinen, V., et al. 2009a, GCN Circ., 9518

Cenko, S. B., Fox, D. B., Moon, D.-S., et al. 2006b, PASP, 118, 1396

Cenko, S. B., Kelemen, J., Harrison, F. A., et al. 2009b, ApJ, 693, 1484

Cenko, S. B., Butler, N. R., Ofek, E. O., et al. 2010, AJ, 140, 224

Chen, H.-W., Helsby, J., Shectman, S., Thompson, I., \& Crane, J. 2009, GCN Circ., 10038

Chen, H.-W., Prochaska, J. X., Herbert-Fort, S., Christlein, D., \& Cortes, S. 2007, GCN Circ., 6217

Chornock, R., Cenko, S. B., Griffith, C. V., et al. 2009a, GCN Circ., 9151

Chornock, R., Foley, R. J., Li, W., \& Filippenko, A. V. 2008, GCN Circ., 7381

Chornock, R., Li, W., \& Filippenko, A. V. 2009b, GCN Circ., 8979

Chornock, R., Perley, D. A., Cenko, S. B., \& Bloom, J. S. 2009c, GCN Circ., 9243

Chornock, R., Perley, D. A., Cenko, S. B., et al. 2009d, GCN Circ., 8994

Chornock, R., Perley, D. A., \& Cobb, B. E. 2009e, GCN Circ., 10100

Covino, S., Melandri, A., Salvaterra, R., et al. 2013, MNRAS, 432, 1231

Coward, D. M., Howell, E. J., Branchesi, M., et al. 2013, MNRAS, 432, 2141 
Cucchiara, A. 2010, GCN Circ., 10473

Cucchiara, A., Fox, D., Levan, A., \& Tanvir, N. 2009, GCN Circ., 10202

Cucchiara, A., \& Fox, D. B. 2008, GCN Circ., 7654

Cucchiara, A., Fox, D. B., Cenko, S. B., \& Berger, E. 2008a, GCN Circ., 8346

Cucchiara, A., Fox, D. B., Cenko, S. B., \& Berger, E. 2008b, GCN Circ., 8713

Cucchiara, A., Levan, A. J., Fox, D. B., et al. 2011, ApJ, 736, 7

Cucchiara, A., Fumagalli, M., Rafelski, M., et al. 2015, ApJ, 804, 51

Daigne, F., Rossi, E. M., \& Mochkovitch, R. 2006, MNRAS, 372, 1034

D’Avanzo, P., D'Elia, V., Covino, S., et al. 2008, GCN Circ., 8528

Davé, R. 2008, MNRAS, 385, 147

De Cia, A., Ledoux, C., Savaglio, S., Schady, P., \& Vreeswijk, P. M. 2013, A\&A, 560, A88

de Ugarte Postigo, A., Gorosabel, J., Fynbo, J. P. U., Wiersema, K., \& Tanvir, N. 2009a, GCN Circ., 9771

de Ugarte Postigo, A., Gorosabel, J., Malesani, D., Fynbo, J. P. U., \& Levan, A. J. 2009b, GCN Circ., 9383

de Ugarte Postigo, A., Jakobsson, P., Malesani, D., et al. 2009c, GCN Circ., 8766

de Ugarte Postigo, A., Goldoni, P., Thöne, C. C., et al. 2009d, GCN Circ., 10042

D'Elia, V. 2011, AN, 332, 272

D'Elia, V., Covino, S., \& D'Avanzo, P. 2008a, GCN Circ., 8438

D'Elia, V., Thöne, C. C., de Ugarte Postigo, A., et al. 2008b, GCN Circ., 8531

Djorgovski, S. G., Frail, D. A., Kulkarni, S. R., et al. 2001, ApJ, 562, 654

Dressler, A., Bigelow, B., Hare, T., et al. 2011, PASP, 123, 288

Eliasdóttir, Á, Fynbo, J. P. U., Hjorth, J., et al. 2009, ApJ, 697, 1725

Elliott, J., Greiner, J., Khochfar, S., et al. 2012, A\&A, 539, A113

Evans, P. A., Beardmore, A. P., Page, K. L., et al. 2009, MNRAS, 397, 1177

Ferrero, P., Klose, S., Kann, D. A., et al. 2009, A\&A, 497, 729

Flores, H., Fynbo, J. P. U., de Ugarte Postigo, A., et al. 2010, GCN Circ., 11317

Foley, R. J., Chen, H.-W., Bloom, J., \& Prochaska, J. X. 2005, GCN Circ., 3483

Fong, W., Berger, E., Chornock, R., et al. 2013, ApJ, 769, 56

Friis, M., De Cia, A., Krühler, T., et al. 2015, MNRAS, 451, 167

Fruchter, A. S., Levan, A. J., Strolger, L., et al. 2006, Natur, 441, 463

Fryer, C. L., \& Heger, A. 2005, ApJ, 623, 302

Fynbo, J. P. U., Malesani, D., Jakobsson, P., \& D’Elia, V. 2009a, GCN Circ., 9947

Fynbo, J. P. U., Watson, D., Thöne, C. C., et al. 2006, Natur, 444, 1047

Fynbo, J. P. U., Jakobsson, P., Prochaska, J. X., et al. 2009b, ApJS, 185, 526

Fynbo, J. U., Jensen, B. L., Gorosabel, J., et al. 2001, A\&A, 369, 373

Gal-Yam, A., Fox, D. B., Price, P. A., et al. 2006, Natur, 444, 1053

Gehrels, N., Chincarini, G., Giommi, P., et al. 2004, ApJ, 611, 1005

Gehrels, N., Norris, J. P., Barthelmy, S. D., et al. 2006, Natur, 444, 1044

Gehrels, N., Barthelmy, S. D., Burrows, D. N., et al. 2008, ApJ, 689, 1161

Goad, M. R., Tyler, L. G., Beardmore, A. P., et al. 2007, A\&A, 476, 1401

Goldoni, P., de Ugarte Postigo, A., \& Fynbo, J. P. U. 2013, GCN Circ., 15571

Goldoni, P., Royer, F., François, P., et al. 2006, Proc. SPIE, 6269, 2

Graham, J. F., \& Fruchter, A. S. 2013, ApJ, 774, 119

Greiner, J., Bornemann, W., Clemens, C., et al. 2008, PASP, 120, 405

Greiner, J., Krühler, T., Klose, S., et al. 2011, A\&A, 526, A30

Greiner, J., Krühler, T., Nardini, M., et al. 2013, A\&A, 560, A70

Greiner, J., Fox, D. B., Schady, P., et al. 2015, ApJ, 809, 76

Groot, P. J., Galama, T. J., van Paradijs, J., et al. 1998, ApJL, 493, L27

Grupe, D., Nousek, J. A., vanden Berk, D. E., et al. 2007, AJ, 133, 2216

Guetta, D., \& Della Valle, M. 2007, ApJL, 657, L73

Guetta, D., \& Piran, T. 2007, JCAP, 7, 3

Guidorzi, C., Mundell, C. G., Smith, R. J., et al. 2006, GCN Circ., 4661

Hao, J.-M., \& Yuan, Y.-F. 2013, ApJ, 772, 42

Hashimoto, T., Ohta, K., Aoki, K., et al. 2010, ApJ, 719, 378

Hashimoto, T., Perley, D. A., Ohta, K., et al. 2015, ApJ, 806, 250

Hirschi, R., Meynet, G., \& Maeder, A. 2005, A\&A, 443, 581

Hjorth, J., Malesani, D., Jakobsson, P., et al. 2012, ApJ, 756, 187

Hogg, D. W., Pahre, M. A., McCarthy, J. K., et al. 1997, MNRAS, 288, 404

Hook, I. M., Jørgensen, I., Allington-Smith, J. R., et al. 2004, PASP, 116, 425

Hopkins, A. M., \& Beacom, J. F. 2006, ApJ, 651, 142

Hunt, L. K., Palazzi, E., Michałowski, M. J., et al. 2014, A\&A, 565, A112

Jakobsson, P., de Ugarte Postigo, A., Gorosabel, J., et al. 2009, GCN Circ., 9797

Jakobsson, P., Hjorth, J., Fynbo, J. P. U., et al. 2004, ApJL, 617, L21

Jakobsson, P., Paraficz, D., Telting, J., et al. 2005, GCN Circ., 4015

Jakobsson, P., Levan, A., Fynbo, J. P. U., et al. 2006, A\&A, 447, 897

Jakobsson, P., Fynbo, J. P. U., Andersen, M. I., et al. 2007, GCN Circ., 6398

Jakobsson, P., Hjorth, J., Malesani, D., et al. 2012, ApJ, 752, 62

Jaunsen, A. O., Rol, E., Watson, D. J., et al. 2008, ApJ, 681, 453
Kann, D. A., Klose, S., Zhang, B., et al. 2010, ApJ, 720, 1513

Kawai, N., Kosugi, G., Aoki, K., et al. 2006, Natur, 440, 184

Kelly, P. L., Filippenko, A. V., Modjaz, M., \& Kocevski, D. 2014, ApJ, 789,23

Kistler, M. D., Yüksel, H., Beacom, J. F., Hopkins, A. M., \& Wyithe, J. S. B. 2009, ApJL, 705, L104

Kistler, M. D., Yüksel, H., Beacom, J. F., \& Stanek, K. Z. 2008, ApJL, 673, L119

Kocevski, D., \& West, A. A. 2011, ApJL, 735, L8

Kohn, S. A., Michałowski, M. J., Bourne, N., et al. 2015, MNRAS, 448, 1494

Krühler, T., Malesani, D., Xu, D., et al. 2013, GCN Circ., 14264

Krühler, T., Schrey, F., Greiner, J., et al. 2008, GCN Circ., 8060

Krühler, T., Greiner, J., Schady, P., et al. 2011, A\&A, 534, A108

Krühler, T., Malesani, D., Milvang-Jensen, B., et al. 2012, ApJ, 758, 46

Krühler, T., Malesani, D., Fynbo, J. P. U., et al. 2015, A\&A, 581, A125

Lamb, D. Q., \& Reichart, D. E. 2000, ApJ, 536, 1

Langer, N., \& Norman, C. A. 2006, ApJL, 638, L63

Le, T., \& Dermer, C. D. 2007, ApJ, 661, 394

Le Floc'h, E., Charmandaris, V., Forrest, W. J., et al. 2006, ApJ, 642, 636

Levan, A. J., Jakobsson, P., Hurkett, C., et al. 2007, MNRAS, 378, 1439

Levesque, E. M., Kewley, L. J., Berger, E., \& Zahid, H. J. 2010, AJ, 140, 1557

Lien, A., Sakamoto, T., Gehrels, N., et al. 2014, ApJ, 783, 24

MacFadyen, A. I., \& Woosley, S. E. 1999, ApJ, 524, 262

Madau, P., \& Dickinson, M. 2014, ARA\&A, 52, 415

Madau, P., Pozzetti, L., \& Dickinson, M. 1998, ApJ, 498, 106

McLean, I. S., Steidel, C. C., Epps, H. W., et al. 2012, Proc. SPIE, 8446, 84460J

Melandri, A., Mundell, C. G., Kobayashi, S., et al. 2008, ApJ, 686, 1209

Melandri, A., Mundell, C. G., Cano, Z., et al. 2009, GCN Circ., 9726

Melandri, A., Sbarufatti, B., D'Avanzo, P., et al. 2012, MNRAS, 421, 1265

Mészáros, P., \& Rees, M. J. 1997, ApJ, 476, 232

Michałowski, M. J., Hjorth, J., Castro Cerón, J. M., \& Watson, D. 2008, ApJ, 672,817

Michałowski, M. J., Kamble, A., Hjorth, J., et al. 2012, ApJ, 755, 85

Milvang-Jensen, B., Goldoni, P., Tanvir, N. R., et al. 2010, GCN Circ., 10876

Milvang-Jensen, B., Fynbo, J. P. U., Malesani, D., et al. 2012, ApJ, 756, 25

Mirabal, N., \& Halpern, J. P. 2006, GCN Circ., 4792

Modjaz, M., Kewley, L., Kirshner, R. P., et al. 2008, AJ, 135, 1136

Morgan, A. N. 2014, PhD thesis, Univ. California

Morgan, A. N., Bloom, J. S., \& Klein, C. R. 2009, GCN Circ., 9635

Morgan, A. N., Perley, D. A., Cenko, S. B., et al. 2014, MNRAS, 440, 1810

Mulchaey, J., \& Berger, E. 2005, GCN Circ., 3114

Mundell, C. G., Smith, R. J., \& Cano, Z. 2011, GCN Circ., 11633

Mundell, C. G., Melandri, A., Gomboc, A., et al. 2006, GCN Circ., 4726

Nysewander, M., Fruchter, A. S., \& Pe'er, A. 2009, ApJ, 701, 824

Oates, S. R., \& Markwardt, C. B. 2008, GCN Circ., 7253

Oke, J. B., Cohen, J. G., Carr, M., et al. 1995, PASP, 107, 375

O'Meara, J., Chen, H.-W., \& Prochaska, J. X. 2010, GCN Circ., 11089

Pavlenko, E., Rumyantsev, V., \& Pozanenko, A. 2009, GCN Circ., 9179

Peek, J. E. G., \& Graves, G. J. 2010, ApJ, 719, 415

Perley, D. A., Prochaska, J. X., Kalas, P., et al. 2009a, GCN Circ., 10272

Perley, D. A., Metzger, B. D., Granot, J., et al. 2009b, ApJ, 696, 1871

Perley, D. A., Cenko, S. B., Bloom, J. S., et al. 2009c, AJ, 138, 1690

Perley, D. A., Bloom, J. S., Klein, C. R., et al. 2010, MNRAS, 406, 2473

Perley, D. A., Morgan, A. N., Updike, A., et al. 2011, AJ, 141, 36

Perley, D. A., Levan, A. J., Tanvir, N. R., et al. 2013, ApJ, 778, 128

Perley, D. A., Perley, R. A., Hjorth, J., et al. 2015a, ApJ, 801, 102

Perley, D. A., Tanvir, N. R., Hjorth, J., et al. 2016b, ApJ, 817, 8

Pian, E., Mazzali, P. A., Masetti, N., et al. 2006, Natur, 442, 1011

Poole, T., Moretti, A., Holland, S. T., et al. 2005, GCN Circ., 3698

Porciani, C., \& Madau, P. 2001, ApJ, 548, 522

Price, P. A. 2006, GCN Circ., 5104

Prochaska, J. X., Chen, H.-W., Dessauges-Zavadsky, M., \& Bloom, J. S. 2007, ApJ, 666, 267

Prochaska, J. X., Sheffer, Y., Perley, D. A., et al. 2009, ApJL, 691, L27

Ramirez-Ruiz, E., Trentham, N., \& Blain, A. W. 2002, MNRAS, 329, 465

Rau, A., Fynbo, J., \& Greiner, J. 2010, GCN Circ., 10350

Reddy, N. A., \& Steidel, C. C. 2009, ApJ, 692, 778

Rinner, C., \& Kugel, F. 2008, GCN Circ., 7670

Robertson, B. E., \& Ellis, R. S. 2012, ApJ, 744, 95

Robertson, B. E., Ellis, R. S., Furlanetto, S. R., \& Dunlop, J. S. 2015, ApJL, 802, L19

Rol, E., Tanvir, N., Mirabal, N., et al. 2007, GCN Circ., 6221

Roming, P. W. A., Kennedy, T. E., Mason, K. O., et al. 2005, SSRv, 120, 95

Rossi, A., Klose, S., Ferrero, P., et al. 2012, A\&A, 545, A77

Ruiz-Velasco, A. E., Swan, H., Troja, E., et al. 2007, ApJ, 669, 1 
Sakamoto, T., Barthelmy, S. D., Baumgartner, W. H., et al. 2011, ApJS, 195, 2 Salvaterra, R., \& Chincarini, G. 2007, ApJL, 656, L49

Salvaterra, R., Della Valle, M., Campana, S., et al. 2009, Natur, 461, 1258

Salvaterra, R., Campana, S., Vergani, S. D., et al. 2012, ApJ, 749, 68

Sari, R., \& Piran, T. 1997, ApJ, 485, 270

Schady, P., Savaglio, S., Müller, T., et al. 2014, A\&A, 570, A52

Schlafly, E. F., \& Finkbeiner, D. P. 2011, ApJ, 737, 103

Schlegel, D. J., Finkbeiner, D. P., \& Davis, M. 1998, ApJ, 500, 525

Schulze, S., Chapman, R., Hjorth, J., et al. 2015, ApJ, 808, 73

Skrutskie, M. F., Cutri, R. M., Stiening, R., et al. 2006, AJ, 131, 1163

Sparre, M., Hartoog, O. E., Krühler, T., et al. 2014, ApJ, 785, 150

Stanek, K. Z., Gnedin, O. Y., Beacom, J. F., et al. 2006, AcA, 56, 333

Svensson, K. M., Levan, A. J., Tanvir, N. R., Fruchter, A. S., \& Strolger, L.-G. 2010, MNRAS, 405, 57

Svensson, K. M., Levan, A. J., Tanvir, N. R., et al. 2012, MNRAS, 421, 25

Tanvir, N. R., Barnard, V. E., Blain, A. W., et al. 2004, MNRAS, 352, 1073

Tanvir, N. R., Fox, D. B., Levan, A. J., et al. 2009, Natur, 461, 1254

Tanvir, N. R., Levan, A. J., Fruchter, A. S., et al. 2012, ApJ, 754, 46

Thöne, C. C., Malesani, D., Levan, A. J., et al. 2009a, GCN Circ., 9403

Thöne, C. C., Jakobsson, P., De Cia, A., et al. 2009b, GCN Circ., 9409

Thöne, C. C., Fynbo, J. P. U., Goldoni, P., et al. 2013, MNRAS, 428, 3590

Totani, T. 1997, ApJL, 486, L71

Trenti, M., Perna, R., Levesque, E. M., Shull, J. M., \& Stocke, J. T. 2012, ApJL, 749, L38

Trenti, M., Perna, R., \& Tacchella, S. 2013, ApJL, 773, L22
Updike, A., Nicuesa, A., Nardini, M., Krühler, T., \& Greiner, J. 2010, GCN Circ., 10874

van den Heuvel, E. P. J., \& Portegies Zwart, S. F. 2013, ApJ, 779, 114

van der Horst, A. J., Kouveliotou, C., Gehrels, N., et al. 2009, ApJ, 699, 1087

Vergani, S. D., Salvaterra, R., Japelj, J., et al. 2015, A\&A, 581, A102

Vernet, J., Dekker, H., D’Odorico, S., et al. 2011, A\&A, 536, A105

Virgili, F. J., Liang, E.-W., \& Zhang, B. 2009, MNRAS, 392, 91

Virgili, F. J., Zhang, B., Nagamine, K., \& Choi, J.-H. 2011, MNRAS, 417, 3025

Wanderman, D., \& Piran, T. 2010, MNRAS, 406, 1944

Wang, F. Y., \& Dai, Z. G. 2011, ApJL, 727, L34

Wiersema, K., Levan, A., Kamble, A., Tanvir, N., \& Malesani, D. 2009, GCN Circ., 9673

Wiersema, K., Savaglio, S., Vreeswijk, P. M., et al. 2007, A\&A, 464, 529

Wijers, R. A. M. J., Bloom, J. S., Bagla, J. S., \& Natarajan, P. 1998, MNRAS, 294, L13

Woosley, S. E., \& Heger, A. 2006, ApJ, 637, 914

Yoon, S.-C., \& Langer, N. 2005, A\&A, 443, 643

Yu, H., Wang, F. Y., Dai, Z. G., \& Cheng, K. S. 2015, ApJS, 218, 13

Yüksel, H., Kistler, M. D., Beacom, J. F., \& Hopkins, A. M. 2008, ApJL, 683, L5

Zafar, T., Watson, D., Elíasdóttir, Á, et al. 2012, ApJ, 753, 82

Zauderer, A., \& Berger, E. 2011, GCN Circ., 12190

Zauderer, B. A., Berger, E., Margutti, R., et al. 2013, ApJ, 767, 161 